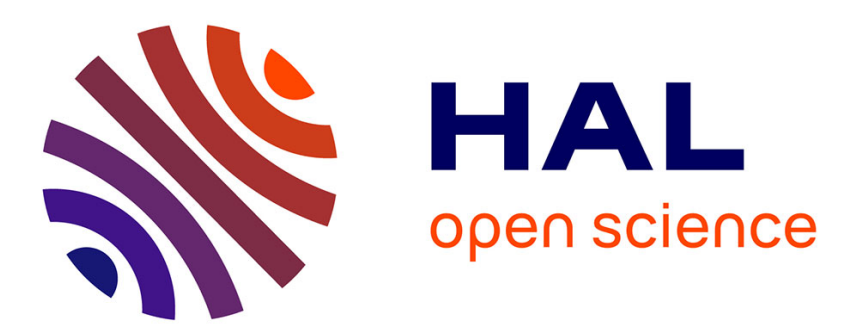

\title{
Impact of particle stiffness on shear-thinning of non-Brownian suspensions
}

Duncan Gilbert, Rudy Valette, Elisabeth Lemaire

\section{To cite this version:}

Duncan Gilbert, Rudy Valette, Elisabeth Lemaire. Impact of particle stiffness on shear-thinning of non-Brownian suspensions. 2021. hal-03353575

\section{HAL Id: hal-03353575 \\ https://hal.science/hal-03353575}

Preprint submitted on 24 Sep 2021

HAL is a multi-disciplinary open access archive for the deposit and dissemination of scientific research documents, whether they are published or not. The documents may come from teaching and research institutions in France or abroad, or from public or private research centers.
L'archive ouverte pluridisciplinaire HAL, est destinée au dépôt et à la diffusion de documents scientifiques de niveau recherche, publiés ou non, émanant des établissements d'enseignement et de recherche français ou étrangers, des laboratoires publics ou privés. 


\section{Impact of particle stiffness on shear-thinning of non-Brownian suspensions}

Duncan Gilbert, ${ }^{1}$ Rudy Valette, ${ }^{2}$ and Elisabeth Lemaire ${ }^{1, \text { a) }}$

${ }^{1)}$ Institut de Physique de Nice, UCA-CNRS UMR 7010, Parc Valrose, 06108 Nice, France

2) PSL Research University, MINES ParisTech, CEMEF, CNRS UMR 7635, CS 10207 rue Claude Daunesse, 06904, Sophia-Antipolis Cedex,

\section{France}

(Dated: 24 September 2021)

We attempt to identify the impact of particle stiffness on the shear-thinning behavior exhibited by frictional and adhesive non-Brownian suspensions. To this aim, we compare the rheological behavior of three suspensions whose particles have different Young's moduli. The stiffest particles are Soda-lime glass beads with a Young modulus of $70 \mathrm{GPa}$. The two other kinds of particles are made of polydimethylsiloxane and have been manufactured using a homemade millifluidic device that allowed us to produce batches of sparsely polydisperse particles with tunable Young modulus: 1.8 MPa for the softest ones and $15 \mathrm{MPa}$ for the stiffest. We show that the observed shear-thinning is mainly caused by the presence of adhesive forces between particles. For each of the three suspensions, the shear-thinning behavior is described by the variation of the jamming volume fraction, $\phi_{m}$, with stress, $\sigma$, and the results are interpreted in the light of the model proposed by [Richards et al., J. Rheol. 64(2), 405-412 (2020)]. We show that the magnitude of variation of $\phi_{m}$ with $\sigma$ is greater the lower the particle stiffness. More precisely, in the adhesive regime, decreasing the particle stiffness leads to a significant decrease in the value of the jamming fraction while, as predicted by the JKR theory, the characteristic adhesion stress, $\sigma_{a}$, does not vary with stiffness. We finally show that, for stresses much higher than $\sigma_{a}$, the suspensions behave like usual frictional suspensions with a viscosity controlled by both the particle volume fraction and the value of the interparticle friction coefficient.

PACS numbers: 82.70.Kj; 62.20.Qp; 47.15.G

a) corresponding author: elemaire@unice.fr 


\section{INTRODUCTION}

Understanding non-Newtonian behaviors of concentrated suspensions has been a long standing problem since particulate flows are involved in many natural or industrial processes. Among them, shear-thinning observed in Brownian colloidal suspensions has retained much interest and the concept of effective volume fraction ${ }^{1,2}$ has proved to be a very efficient approach to capture shear-thinning observed in many systems (blood ${ }^{3}$, food rheology ${ }^{4}$, nanofluids ${ }^{5}$, to cite but a few). Shear-thinning is also sometimes observed in non-Brownian suspensions that are also deemed to be non-colloidal. These observations have raised many questions especially because, for a long time, it was thought that the flow of hard particle suspensions (with no colloidal interactions) was governed only by hydrodynamic interactions between particles. In this framework, as argued by several authors, dimensional analysis results in a Newtonian behavior of a suspension of hard spherical particles dispersed in a Newtonian fluid. This apparent paradox has motivated a number of studies during the last decades. Acrivos et al. ${ }^{6}$ showed that apparent shear-thinning could originate from a density mismatch between the particles and the suspending liquid. As pointed out by the authors themselves, this explanation can be relevant to account for shear-thinning in a Couette flow but cannot provide any explanation of the shear-thinning that is also sometimes observed in other flow geometries (parallel plate or cone plate geometry) ${ }^{7-10}$. Vazquez-Quesada et $a l .{ }^{7,8}$ proposed that, in some cases, the suspension shear-thinning was caused by the non Newtonian behavior of the suspending liquid itself. Even though the suspending liquid is deemed to be Newtonian for moderate shear rates, it may exhibit non-Newtonian characteristics in the range of the very high shear rates within inter-particle gap, which results in an overall shear-thinning of the suspension. Shear rate dependent slip of solvent molecules on particle surfaces has also been invoked to explain shear thinning ${ }^{11}$. Adhesion between particles, which can be accompanied by particle clustering, has also been identified as a possible cause of shear-thinning. Adhesion can act as a rolling constraint that hinders relative particle motion and, as the shear stress increases, the number of adhesive contacts decreases causing a viscosity drop ${ }^{12}$. Adhesion can also be responsible for particle clustering which leads to an effective solid fraction higher than the particle volume fraction because of immobilized liquid trapped inside the aggregates ${ }^{13}$. A last possible cause of shear-thinning recently pointed out, is the decrease of the interparticle friction coefficient when the stress increases. For some years now, experimental evidence was produced to show that solid contacts do exist in sheared $\mathrm{NBS}^{14,15}$ and several numerical studies showed that these contacts, 
especially frictional contacts, have a significant impact on the rheology (viscosity values ${ }^{16-18}$, non Newtonian behaviors ${ }^{19-22}$, normal stress differences ${ }^{23}$, shear-induced diffusion ${ }^{24} \ldots$ ) of concentrated non-Brownian suspensions. In the shear-thinning scenario proposed by Lobry et al. ${ }^{21}$, the contact between one or a few asperities is considered. For such a contact, the value of the friction coefficient is expected to decrease with normal force ${ }^{25,26}$ and thus with shear stress. By introducing such a tangential contact law in numerical simulations, the authors showed a decrease of the viscosity with shear stress that is in the same range as that observed experimentally. Recently, Papadopoulou et $a l^{27}$ showed that depending on the interactions between the suspending fluid and the particles, either of the two last mechanisms (adhesion or load variable friction) can explain shear-thinning.

All the studies mentioned above deal with rigid particles. The rheology of non-Brownian suspensions of deformable particles is much less well documented ( $\operatorname{se}^{28}$ for a review). Two studies ${ }^{29,30}$ have investigated the influence of particle rigidity on the rheology of aqueous suspensions of agarose particles. By manufacturing particles with different agar concentrations (comprised between 0.5 and $5 w t \%$ ), the authors changed the elastic modulus of the particles in the range $2.4-185 \mathrm{kPa}$. A shear-thinning for the suspensions composed of the softest particles is observed while the suspensions of stiffer particles exhibit an almost Newtonian behavior. The viscosity is shown to decrease with decreasing elastic modulus of the particles, especially under high shear stress. This result is in agreement with the model proposed by Snabre $\&$ Mills ${ }^{31}$ and is explained by the increased deformability of the softer particles allowing greater rearrangement to be achieved.

Here, we will study the impact of particle stiffness on the suspension rheology in a different range of elastic moduli. We chose particles whose elastic modulus is of the order of $10 \mathrm{MPa}$, thus about 1000 times softer than the usual rigid particles (PMMA, PS, glass...) but stiff enough not to be deformed by the flow. The deformability of the particles can be quantified by the capillary number $C a=\sigma / E$ where $\sigma$ is the shear stress and $E$ the elastic modulus of the particles, low $C a$ values meaning that particles are hardly deformed by the flow. In the current study, the maximum capillary number will be of the order of $10^{-4}$, so that no significant deformation at the particle scale is expected. However we will show that, even for such small capillary number values, the contact properties (adhesion and friction) vary with particle stiffness, resulting in a significant alteration of the rheological behavior of the suspensions. To this aim, we will compare the rheological behavior of three non-Brownian suspensions, one consisting of silica particles of $90 \mu \mathrm{m}$ 
diameter dispersed in a sodium polytungstate aqueous solution (TC-Tungsten Coumpounds), the two others of polydimethylsiloxane (PDMS) particles of $110 \mu \mathrm{m}$ in diameter and Young's modulus of $1.8 \mathrm{MPa}$ for the softest ones and of $15 \mathrm{MPa}$ for the stiffest ones, dispersed in Span 80 (Sorbitan monooleate, Alfa Aesar). In Sec. II, we discuss the possible causes of shear-thinning in non-Brownian suspensions and present the different models of the literature that allow linking shear-thinning to interparticle contact properties and suspension microstructure. In the following section (Sec. III), we describe and characterize the device that makes it possible to manufacture the PDMS particles and we try to extensively characterize them. Section IV is devoted to the description of the rheometry experiments whose results are reported and discussed in Sec. V.

\section{SHEAR-THINNING IN NON-BROWNIAN SUSPENSIONS}

In this section, we examine two of the mechanisms proposed to explain the shear-thinning behavior of non-Brownian suspensions: the presence of adhesive forces between particles and the existence of a stress-dependent inter-particle friction coefficient. As proposed by many authors, it is possible to capture the non-Newtonian behavior observed in non-Brownian suspensions by introducing a jamming fraction, $\phi_{m}$, that is a function of the shear stress. Thus, in the case of shearthickening suspensions, the jamming fraction decreases as the stress increases ${ }^{17,32,33}$, while in the case of shear-thinning suspensions, $\phi_{m}$ increases as the stress grows ${ }^{13,21,34,35}$. In the following, we will adopt this approach and characterize shear-thinning observed in several types of suspensions by the variation of $\phi_{m}$ with $\sigma$. We will see in the following that this choice makes it possible to present the rheology results in a compact form which facilitates the comparison of the behavior of different systems but also enables, in a certain number of cases, to rule on the cause of shearthinning.

\section{A. Role of adhesive forces}

As early as 1995 , Zhou et al. ${ }^{13}$ proposed to capture the shear-thinning observed in micronic non-Brownian sphere suspensions by introducing a jamming fraction that varies with shear stress:

$$
\eta_{S}=\left(\frac{1}{1-\frac{\phi}{\phi_{m}}}\right)^{[\eta] \phi_{m}}
$$


where $\eta_{S}=\eta / \eta_{0}$ is the viscosity of the suspension normalized by the suspending fluid viscosity. $[\eta]$ is the intrinsic viscosity and $\phi_{m}=\phi_{m}(\sigma)$ is the jamming fraction that is assumed to vary with shear stress. The relation between $\phi_{m}$ and $\sigma$ is deduced from a microstructural model where the aggregation state of a concentrated suspension is controlled by the balance between adhesive forces that lead particles to form aggregates and flow-induced forces that tend to break the microstructure:

$$
\frac{1}{\phi_{m}}=\frac{1}{\phi_{m}^{\infty}}+\left(\frac{1}{\phi_{m}^{0}}-\frac{1}{\phi_{m}^{\infty}}\right) \lambda
$$

where $\phi_{m}^{0}$ and $\phi_{m}^{\infty}$ are the limiting jamming fractions that correspond respectively to the low-shear regime where particles are form clusters and to the high-shear regime where flow-induced forces are dominant. $\lambda=\left(1+\frac{\sigma}{\sigma_{a}}\right)^{-p}$ is a structural parameter that defines the proportion of particles belonging to aggregates. $\sigma_{a}$ is the characteristic stress of adhesive contact rupture and $p$ a fitting parameter of the order of 1 that represents the sensitivity of the microstructure to the shear stress.

This model enabled to represent the shear-thinning observed in a variety of suspensions composed of particles of different nature (Silica, several metallic oxides, cocoa powder) and size (ranging from typically 1 to $10 \mu \mathrm{m}$ ), suspended in different media (glycerol, water, cocoa fat) and for particle volume fractions covering the range from a few percent to more than $60 \%{ }^{13}$. Depending on the suspension composition, $\sigma_{a}$ can take values between a few Pascals and a few tens of Pascals. These values are of the same order of magnitude as the stress used to characterize the rheology of suspensions, so that adhesion between particles has a significant influence on the flow properties of the suspensions.

More recently, Guy et al. ${ }^{36}$ proposed another phenomenological model based on the idea that particle interactions -whether adhesive or frictional- act as constraints that hinder relative particle motion. Richards et al. ${ }^{12}$ used this model to study the effect of adhesion in frictional suspensions and proposed that the jamming volume fraction (and therefore the viscosity) is controlled by the proportion of adhesive contacts in the suspension, which itself depends on the shear stress value:

$$
\phi_{m}=\phi_{\mu}+\left(\phi_{a l p}-\phi_{\mu}\right) a(\sigma)
$$

$\phi_{a l p}$ is the jamming fraction at $\sigma \rightarrow 0$ for a suspension where all contacts are adhesive. Its value is not well known but Richards et al. proposed to take as an order of magnitude the value of loose packing for adhesive suspensions, hence its name, for which some numerical studies have given an estimate between 0.15 and $0.51^{37-39} . \phi_{\mu}$ is the jamming volume fraction for a non-adhesive 
frictional suspension whose value depends on the friction coefficient between particles and is now well documented $16,18,20,21,40$.

Finally, Richards et al. proposed the following expression for the variation of the fraction of adhesive contacts with shear stress ${ }^{12}$ :

$$
a(\sigma)=1-\exp \left[-\left(\frac{\sigma_{a}}{\sigma}\right)^{\kappa}\right]
$$

$\sigma_{a}$ is, as in the model of Zhou et al., the characteristic adhesive stress and $\kappa$ a parameter that describes how rapidly adhesive contacts break under the action of shear stress.

\section{B. Shear-thinning explained by a stress-dependent interparticle friction coefficient}

Quite recently, Chatté et al. ${ }^{41}$ showed that the shear-thinning which takes place for higher stresses than the DST characteristic stress, i.e. when the contacts have become frictional, was correlated to the decrease of the friction coefficient of the particles as the contact normal force between particles increases. This decrease of the friction coefficient is shown to be correlated to the decrease in the viscosity of the suspension. Besides this first experimental evidence, Tanner $e t$ $a l .{ }^{42}$ proposed a model based on a bootstrap mechanism of friction where the friction coefficient is a decreasing function of the sliding speed between particles and Lobry et al. ${ }^{21}$ showed that it was possible to quantitatively capture the shear-thinning behavior of a non-adhesive non-Brownian suspension of polystyrene particles by properly modeling the contact between particles. This model is based on the description of an interparticle contact which involves only one or a few asperities present on the surface of the particles. In this case, the contact cannot be modeled by Coulomb's law -which involves a constant coefficient- but it is necessary to take into account the variation of the contact area when the normal force varies. Lobry et al. use the elastoplastic contact model proposed by Brizmer et al. ${ }^{26}$ to describe the contact between a sphere and a plane, both perfectly smooth. As long as the contact is elastic, the contact area obeys Hertz's law according to which the contact area varies as the normal force to the power $2 / 3$. Since the tangential force in sliding contact is proportional to the contact area, the friction coefficient that is given by the ratio of the tangential force to the normal force varies as the normal force to the power of $(-1 / 3)$. For higher loads, the contact enters the plastic regime, and the friction coefficient levels off at a constant value. The force experienced by particles in a shear flow is of the order of:

$$
F_{n} \approx 6 \pi a^{2} \sigma
$$


$a$ being the particle radius. Thus it is expected that for "small" shear stresses, $\mu \propto \sigma^{-1 / 3}$ and levels off at "high" shear stress. Moreover, it has been well-established that the viscosity of non-Brownian suspensions increases with increasing inter-particle friction coefficient ${ }^{16-18,21}$. It follows that viscosity decreases in the elastic regime and tends towards a limit value when the plastic contact regime is reached. The transition from the elastic to the plastic regime occurs at a characteristic stress given by ${ }^{21,26}$ :

$$
\sigma_{c}=\pi^{2}(1.234+1.25 v)^{3} \bar{\sigma}_{c} \frac{Y_{0}}{9}\left(\frac{h_{r}}{a} \frac{\left(1-v^{2}\right) Y_{0}}{E}\right)^{2}
$$

where $\bar{\sigma}_{c}=8.88 v-10.13\left(v^{2}+0.089\right), v$ being the Poisson's ratio, $Y_{0}$ and $E$, the yield strength and the Young's modulus of the material constituting the particles and $h_{r}$, the radius of curvature of the contacting zone.

\section{Some features of shear-thinning caused either by adhesive forces or by variable friction coefficient}

The scenario according to which shear-thinning comes from the existence of a friction coefficient that varies with shear stress leads to a variation of $\phi_{m}$ in a rather small range of values. Singh et al. ${ }^{17}$ and Lobry et al. ${ }^{21}$ have proposed empirical relationships between $\phi_{m}$ and $\mu$ deduced from numerical simulations. The variation of viscosity with particle volume fraction is fitted with

a Maron-Pierce like law: $\eta_{S} \propto\left(1-\phi / \phi_{m}\right)^{-2}$ which results in values of $\phi_{m}(\mu)$ that are slightly different in the two studies but give about the same range of variation of $\phi_{m}$ when $\mu$ varies from 0 to 10: $\phi_{m} \in[0.564 ; 0.646]$ for Singh et al. and $\phi_{m} \in[0.551 ; 0.700]$ for Lobry et al. Note that Chèvremont et al. ${ }^{18}$ also proposed a relationship between $\phi_{m}$ and $\mu$ which gives results roughly equivalent to those of Singh et al. and Lobry et al. while using a different constitutive law to relate viscosity to particle volume fraction. All these numerical results show that, in the case of non-adhesive and non-Brownian suspensions, the jamming volume fraction decreases with $\mu$ but can never be lower than typically 0.5. Thus, if lower values of $\phi_{m}$ are observed at low stresses, it would suggest that shear-thinning stems from another mechanism than the reduction of friction coefficient when increasing shear stress. Moreover, in the model of shear-thinning caused by variable friction, the characteristic stress $\sigma_{c}$ which sets the stress scale can be directly evaluated from the material characteristics of the particles. As a consequence, if shear-thinning is observed for $\sigma>>\sigma_{c}$, again, the scenario proposed by Lobry et al. is not appropriate and shear thinning should 
result from adhesive forces.

In the following, we will present the shear-thinning behavior displayed by the three kinds of suspensions and, for each of them, we will use the above criteria to the assess the origin of shearthinning.

\section{MATERIALS}

\section{A. Manufacturing of soft particles}

\section{PDMS particles}

Since to the best of our knowledge, there are no commercially available PDMS particles, we decided to manufacture them. PDMS droplets are produced in a co-flow milli-fluidic device in the jetting regime which ensures a larger production rate than the dripping regime. Our device is quite similar to the one proposed by Carneiro et al. to fabricate PDMS particles of a few tens of microns in diameter ${ }^{43}$. The choice of PDMS as soft material has been guided by several factors. First, PDMS properties are well known due to its widespread use in microfluidics and biotechnology to cite but a few examples. Second, PDMS offers a broad choice of elastic moduli and is easy to shape since it becomes solid by adding a curing agent. At last, PDMS is quite a perfectly elastic material over a large deformation range which ensures mechanical reversibility. We opted for Sylgard 184 (Dowfax 184, DOWCorning) that is commonly used in microfluidics and whose properties are well known. Sylgard 184 has the advantage of having a rather low viscosity (about $3 P a . s$ ) and a fairly low modulus of rigidity, of the order of $2 M P a$, after curing. Sylgard 184 is supplied as a two-part liquid component kit: a prepolymer (part A) and a cross-linking curing agent (part B). When the two parts are mixed at a mixing ratio of $(10: 1)$, the cure reaction begins but the pot life is rather long (about $48 h$ at room temperature), which offers the possibility to keep the PDMS in liquid form for a long enough time and to manufacture particles. The curing time can be shortened by heating the mixture and, for instance, decreases to $35 \mathrm{~min}$ at $T=100^{\circ} \mathrm{C}$. As a matter of fact, we will use this property to produce microparticles.

We manufactured two kinds of particles with two different elastic moduli. The first ones are made exclusively of Sylgard 184 with a 10:1 mixing ratio, hereafter referred to as "soft particles". The viscosity of the PDMS mixture has been measured to be 3 Pa.s at room temperature and the elastic modulus of the cross-linked PDMS to be 1.8MPa. To obtain the second kind of particles, 
hereafter named "stiff particles", toluene was added to Sylgard 184 (10:1 mixing ratio). Toluene is a good solvent for PDMS and allows the chains to swell making the cross-linking points more easily accessible, which increases the cross-linking density and therefore the elastic modulus of the cross-linked PDMS. By adding 5\% toluene, the viscosity of the mixture PDMS/toluene decreases to 1.2 Pa.s while particle stiffness increases to $15 \mathrm{MPa}$. The whole characterization of the particles will be given in Sec III C.

\section{Manufacturing device}

The microparticles are manufactured using a very simple millifluidic device (see Fig. 1) that is designed to create PDMS droplets that are then cured to form spherical elastic particles. To this aim, a co-flow geometry is used where the liquid PDMS is injected through a thin fused silica capillary $($ Molex, $L L C$, inner diameter $650 \mu \mathrm{m})$ into a larger channel where a mixture of 95\% glycerin (Droguerie JARY) and 5\% deionized water is flowing under a controlled pressure gradient. The viscosity of the water/glycerin mixture is 0.5 Pa.s. The PDMS flow rate is controlled by using a syringe pump (Harvard Apparatus PHD2000) and is set to a value comprised between 0.25 and $2 \mathrm{~mL} / \mathrm{h}$. The outer channel is machined in a PMMA block and has a diameter of $1 \mathrm{~mm}$ and a length of $70 \mathrm{~mm}$. The pressure drop across the channel is set to $1 \mathrm{Bar}$, which corresponds to a flow rate of about $130 \mathrm{~mL} / \mathrm{h}$.

The glycerin flow stretches the PDMS jet that destabilizes a few centimeters after the exit of the thin capillary into droplets that are conveyed through a circular channel $(5 \mathrm{~mm}$ in diameter, $60 \mathrm{~cm}$ in length) up to the bottom of a heated beaker that contains an aqueous solution of sodium dodecyl sulfate (SDS) with a concentration of $2 \mathrm{~g} / \mathrm{L}$. The temperature of the beaker is kept at $80^{\circ} \mathrm{C}$, which allows a rapid curing of the droplets.

When the jet containing the droplets reaches the bottom of the beaker, the particles that are less dense than the surrounding glycerin begin to rise up to the diffuse water/glycerin interface. This may appear to be an insignificant detail but is yet crucial for obtaining particles. Indeed, if the particles reach the air/liquid interface, they coalesce under the effect of interfacial tension forces that deform the interface. On the contrary, their residence at the diffuse water/glycerin interface and the presence of SDS in solution enable them to remain spherical and well dispersed throughout their curing. The beaker containing the particles is kept at $T=80^{\circ} \mathrm{C}$ for $12 \mathrm{~h}$. Then the particles are filtered, washed with deionized water, and dried at $60^{\circ} \mathrm{C}$ for $2 h$ in a vacuum drying oven. 


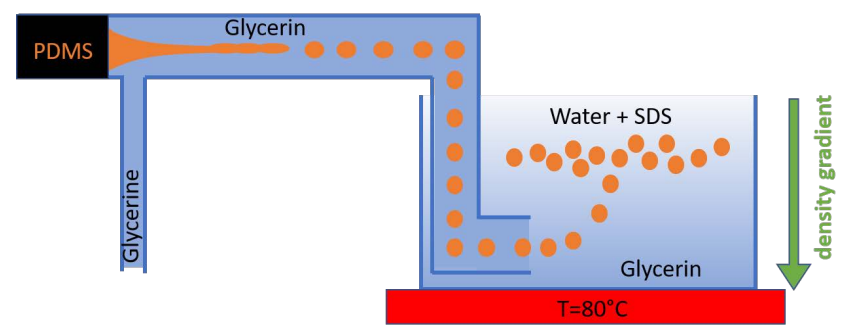

FIG. 1. Schematic view of the device used for the production of controlled-size PDMS particles.

The size of the droplets is directly related to the jet diameter which itself is fixed by the ratio of the flow rates of PDMS, $Q_{i}$ and glycerin, $Q_{e}$. A lower bound estimate of the jet diameter, $d$ can be obtained by assuming that the jet velocity is the same as that which glycerin would have had in the absence of PDMS:

$$
d^{2}=\frac{Q_{i}}{Q_{e}} \frac{D^{2}}{2}
$$

where $D$ is the diameter of the external capillary.

Then the droplet diameter is given by:

$$
2 a=\left(\frac{3}{2} \frac{\lambda}{2 r}\right)^{1 / 3} d
$$

where $\lambda$ is the wavelength of the varicosity of the jet. $\lambda / d$ is of the order of 5 and weakly depends on the viscosity ratio of the fluids inside and outside the jet. For a viscosity ratio of 6 , an estimate of $\lambda / d \approx 6.7$ is found $\mathrm{in}^{44}$.

The variation of the particle size with the square root of the flow rate ratio is displayed in Fig. 2 where is also represented the droplet size estimated from Eqs. (7-8). As can be observed, the droplets produced with the millifluidic device are slightly larger than expected from Eqs.(7-8). This difference is likely to be explained by the probable misalignment of the inner capillary which reduces the effective stretching velocity of the jet. Despite this difference, a linear variation of the particle size with the square root of the flow rate ratio is observed and particles with reproducible and predictable size can be produced with this simple device.

Fig. 3 shows the size distributions measured from image analysis for four flow rate ratios. For low PDMS flow rates, the particle size distribution is narrow and the average diameter matches our target that is to manufacture particles of a hundred micron in diameter. By increasing the flow rate of PDMS, the mean particle size increases and the size distribution becomes broader. Note that we did not notice any shrinkage of the particles during curing which is consistent with 


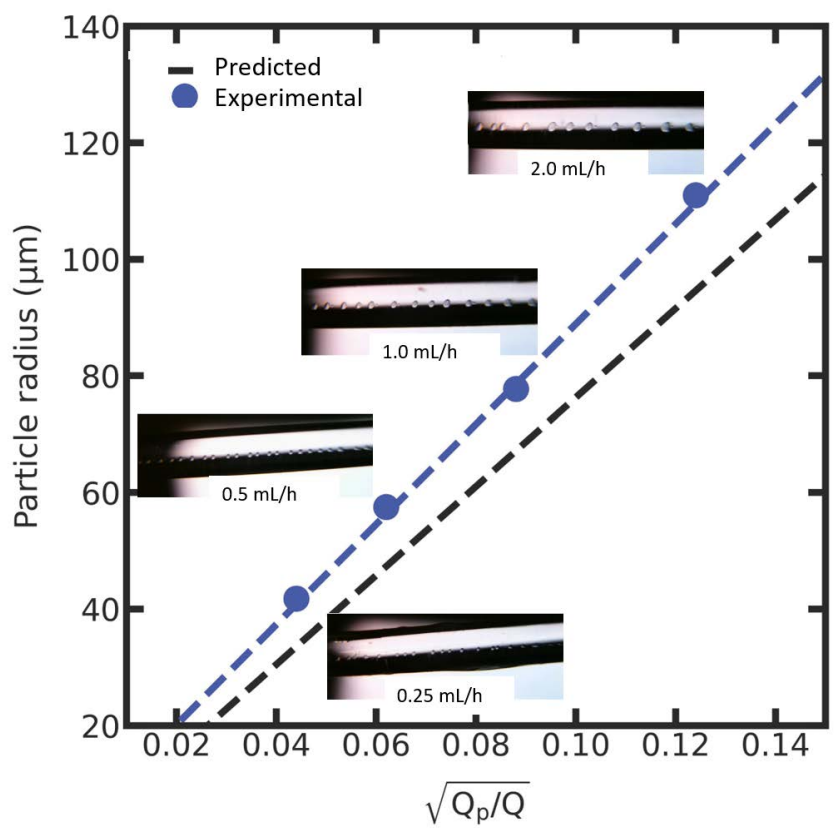

FIG. 2. Variation of particle diameter with the square root of the flow rate ratio. The glycerin flow rate is fixed to $130 \mathrm{~mL} / \mathrm{h}$ and the PDMS flow rate is varied from 0.2 to $2 \mathrm{~mL} / \mathrm{h}$. The difference between the sizes predicted by Eq. (7-8) and the measurements is explained by a slight misalignment of the inner capillary.

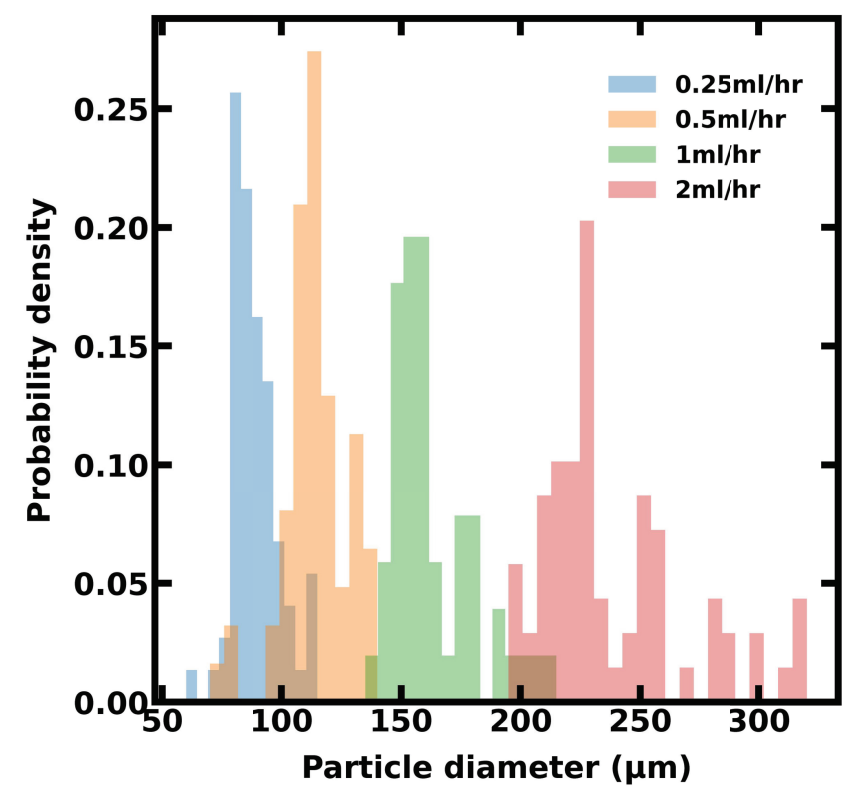

FIG. 3. Number particle size distribution measured for 4 PDMS flow rates. The glycerin flow rate is fixed to $130 \mathrm{~mL} / \mathrm{h}$. 
the observation of Carneiro et al. ${ }^{43}$ who measured both size distributions of either the droplets or the PDMS particles and obtained quite similar distributions but contrasts with the very significant shrinkage reported in ${ }^{45}$.

\section{B. Suspensions}

\section{Suspensions of PDMS particles}

To remove the uncrosslinked oligomers on the surface of PDMS, the particles are washed with acetone. Then, after drying, they are dispersed in Span 80 that is usually used as a W/O surfactant but is used here as the suspending liquid. We made this choice because of the good wetting properties of Span 80 on PDMS surfaces ${ }^{46}$.

The contact angle of Span 80 on a PDMS smooth surface has been measured using a DSA30 goniometer (Bruker) and has been found to be $\theta_{c}=45 \pm 1.5^{\circ}$ for both "soft" and "stiff" PDMS. The superficial tension of Span 80 has been measured to be $\gamma_{l v}=29.2 \pm 0.8 \mathrm{~mJ} / \mathrm{m}^{2}$, using both the Wilhelmy plate method and the drop pending method. Furthermore, the density of Span 80 has been measured to be $0.985 \mathrm{~g} / \mathrm{cm}^{3}$, which is close to PDMS density and enables to obtain nonbuoyant suspensions. At last, the viscosity of Span 80 has been measured to be independent of the shear rate and to be equal to $1.46 \pm 0.03$ Pa.s at $T=22^{\circ} \mathrm{C}$.

\section{Suspensions of Silica particles}

In order to investigate the influence of Young modulus of the particles on suspension rheology, we will compare the rheological behavior of the two types of suspensions composed of PDMS particles dispersed in Span 80 to that of suspensions composed of glass beads of equivalent diameter dispersed in a Newtonian fluid. The glass beads (Soda-lime glass beads, Potters Industries

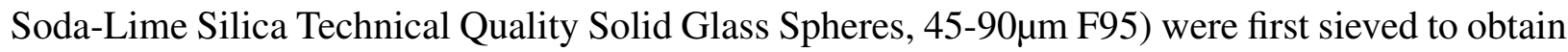
particle size in the range of $80 \mu \mathrm{m}$ to $100 \mu \mathrm{m}$. The difficulty of making glass bead suspensions with a particle diameter close to $100 \mu \mathrm{m}$ lies in the very high density of glass $\left(\sim 2.3 \mathrm{~g} / \mathrm{cm}^{3}\right)$, which is much larger than that of usual liquids. We have chosen to disperse them in a solution of $49.7 \mathrm{wt} \%$ Sodium polytungstate (STP-1, TC-Tungsten Compounds in a mixture of $31.1 \mathrm{wt} \%$ deionized water and $19.2 w t \%$ Ucon oil $(75-H-90,000$, Dow $)$. This solution is a Newtonian fluid of viscosity 0.19 Pa.s at $T=10^{\circ} \mathrm{C}$ and density $2.0 \mathrm{~g} / \mathrm{cm}^{3}$. Note that we chose to work at $T=10^{\circ} \mathrm{C}$ rather than 
at room temperature in order to limit water evaporation. The effects of gravity can most of the time be neglected. For this to be the case, it is necessary that the Shields number, constructed as the ratio between the viscous force exerted on a particle to its apparent weight, is larger than one:

$$
S h=\frac{9}{2} \frac{\sigma}{\Delta \rho g a} \gtrsim 1
$$

Therefore, this condition requires the shear stress to be greater than $3.10^{-2} \mathrm{~Pa}$.

\section{PDMS particle characterization}

\section{Size and density}

We aim at studying the rheological behavior of non-Brownian suspensions. Thus, particles have to be large enough so that Péclet number is much greater than unity, including for low shear rates:

$$
P e=\frac{6 \pi \eta_{0} \dot{\gamma} a^{3}}{k_{B} T}
$$

where $\eta_{0}$ is the viscosity of the suspending fluid, $\dot{\gamma}$, the shear rate, a, the particle radius and $k_{B}=$ 1.34.10 ${ }^{-23} \mathrm{~m}^{2} \mathrm{~kg} \mathrm{~s}^{-2} \mathrm{~K}^{-1}$, the Boltzman constant. On the other hand, it is difficult to make reliable viscosity measurements of suspensions with particle sizes larger than a few hundred microns. Indeed, for viscosity measurement to be independent of the typical scale of the shear flow size, $h$, it is necessary that $h / a \gtrsim 30-40^{47}$. Therefore, particles of a hundred microns are a good compromise. An illustration of the particles produced by the method depicted in Sec. III A 2 is given in Fig. 4 where the presence of very small particles (with typical diameters smaller or of the order of $10 \mu \mathrm{m}$ ) is observed. They come from the jet destabilization that generates satellite drops ${ }^{48}$ that are very difficult to avoid. Nevertheless, they represent a very small volume and would not affect the rheological behavior of the suspensions.

The size distribution histograms for "soft" and "stiff" PDMS particles are shown in Fig. 5. The mean particle diameters are respectively $104 \pm 8 \mu \mathrm{m}$ and $115 \pm 10 \mu \mathrm{m}$.

The particle density is measured with a liquid picnometer filled with Span 80 and is found to be $1.055 \mathrm{~g} / \mathrm{cm}^{3}$ and $1.090 \mathrm{~g} / \mathrm{cm}^{3}$ for "soft" and "stiff" PDMS particles, respectively. 


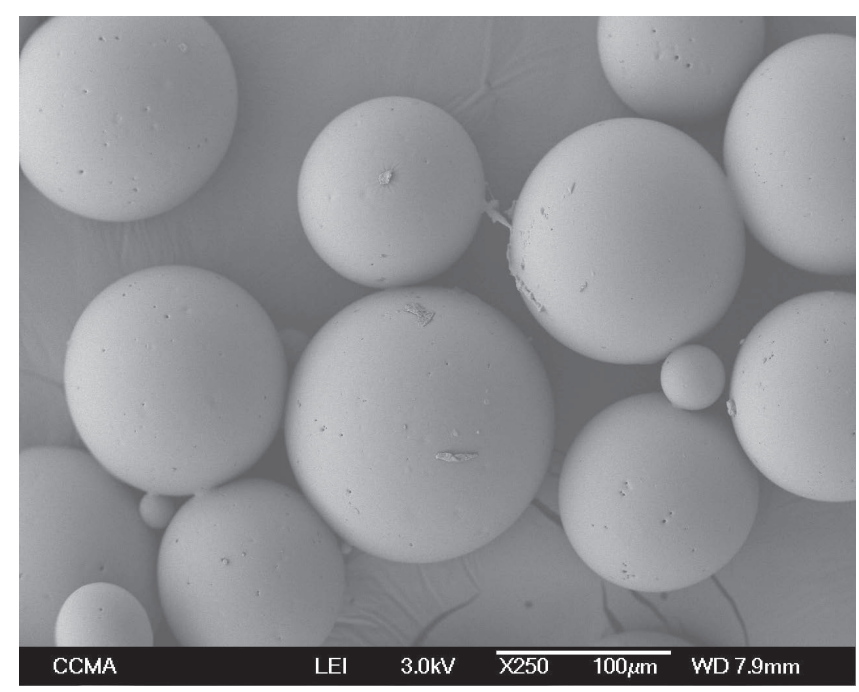

FIG. 4. Electron micrograph of manufactured stiff PDMS particles.

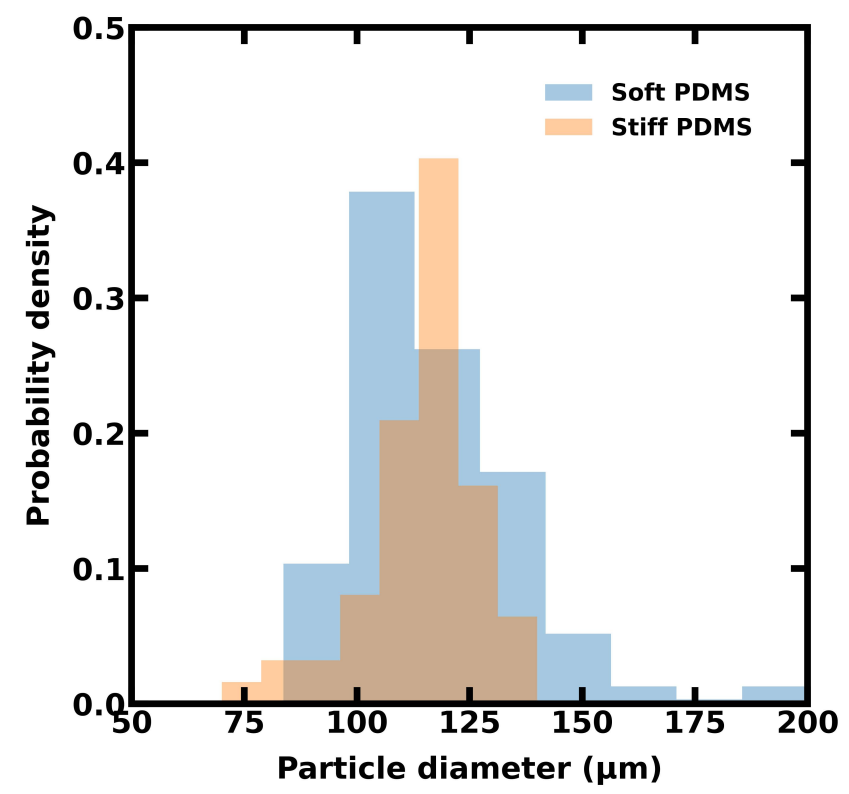

FIG. 5. Number size distribution measured for soft (blue) and stiff (orange) PDMS particles.

\section{Elastic modulus}

It is important to remember that the PDMS mechanical properties depend on the processing conditions, including curing temperature ${ }^{49}$ and washing procedure ${ }^{50,51}$. Therefore, we measured the Young modulus of both kinds of particles by using a nano-indenter (nano-indenter Chiaro, Optics 1 life, radius of the indenting sphere: $9 \mu \mathrm{m}$ ). We obtained $E_{1}=1.8 \pm 0.2 M P a$ for the softer particles and $E_{2}=15 \pm 2 M P a$ for the stiffer ones. 


\section{Evaluation of the inter-particle contact properties}

The purpose of this section is not to present an exhaustive characterization of the contact properties (adhesion, friction) of the particles but only to show that the knowledge of the mechanical and physicochemical properties of the particles makes it possible to propose an order of magnitude for the adhesive force and a scaling law for the inter-particle friction involved in the suspensions of PDMS particles.

The adhesion properties of PDMS have been studied by many authors ${ }^{52-56}$ who all found a value between 40 and $50 \mathrm{~mJ} / \mathrm{m}^{2}$ for the work of adhesion in air. In this range, the most commonly accepted value seems to be $w_{a}=44 \mathrm{~mJ} / \mathrm{m}^{2}$, as pointed out by Oláh \& Vancso ${ }^{57}$. This is in particular the value given by Chaudhury \& Whitesides ${ }^{58}$ who measured the adhesion energy using two different methods, one based on the study of the deformation resulting from the contact between a hemispherical lens and a flat sheet of PDMS and the second based on detailed contact angle measurements with various liquids. For the latter measurements, Chaudhury \& Whitesides ${ }^{58}$ used the Good-Girifalco-Fowkes equation ${ }^{59-61}$ that relates the surface free energy of a solid, $\gamma_{s v}=w_{a} / 2$, to the interfacial tension, $\gamma_{l v}$, and the contact angle, $\theta_{c}$, of non polar fluids:

$$
\gamma_{s v}=\gamma_{l v} \frac{\left(1+\cos \theta_{c}\right)^{2}}{4}
$$

This equation is valid if the surface free energy of the solid results mainly from dispersive forces which is the case of PDMS surfaces and thus provides a way to evaluate $w_{a}$. Taking $\gamma_{l v}=$ $29.2 \pm 0.8 \mathrm{~mJ} / \mathrm{m}^{2}$ for the interfacial tension of Span 80 and $\theta_{c}=45 \pm 1.5^{\circ}$ for the contact angle between Span 80 and PDMS, we obtain $w_{a} \approx 42.6 \pm 2 \mathrm{~mJ} / \mathrm{m}^{2}$, a value in the range of the values found in literature. Then, to have an estimate of the value of the work of adhesion for PDMS in Span $80, w_{S}$, the Young-Dupré relation can be used:

$$
w_{S}=w_{a}-2 \gamma_{l v} \cos \theta_{c}
$$

which gives $w_{S} \approx 1.25 \pm 0.2 \mathrm{~mJ} / \mathrm{m}^{2}$

This value is probably underestimated because the use of the Good-Girifalco-Fowkes (Eq. 11) supposes that the adhesive forces are purely dispersive and that the contacting liquid is nonpolar ${ }^{58}$ whereas Span 80 is known to be weakly polar. As a consequence, other interactions than purely dispersive forces may contribute (weakly) to the adhesion energy of PDMS in Span 80. This estimate obtain for the "soft" PDMS should also be valid for the "hard" PDMS since it has been 
shown, in particular by varying the mixing ratio from 10:1 to 50:1 in Sylgard 184, that the surface energy hardly varies with cross-linking density ${ }^{54}$. Moreover, we did not notice any significant difference in the value of the contact angles between the two types of PDMS (see Sec. III B). We can therefore reasonably take the same estimate of $w_{S}$ for both kinds of particles.

According to JKR theory, the adhesion force, defined as the minimum force required to separate two contacting spheres, is given by ${ }^{62}$ :

$$
F_{a d h}=\frac{3}{4} w_{S} \pi a
$$

Thus, as per JKR theory, the adhesive force does not depend upon the elastic modulus of the particles but only on the surface energy from which we deduce $F_{a d h} \approx 160 n N$ for both kinds of PDMS particle suspensions, which gives a characteristic adhesive stress $\sigma_{a d h}=\frac{F_{a d h}}{6 \pi a^{2}} \sim 3 P a$.

It is anticipated that the frictional properties of the PDMS particles would be quite unusual. Indeed, the PDMS particles that compose the suspensions we study have several untypical characteristics: they are smooth, soft and fully elastic while archetypical particles (PMMA, PS, glass particles...) that usually compose non-Brownian suspensions are rough and hard and become plastic for rather low stress levels. The smoothness of the PDMS particles is ensured by the manufacturing process that minimizes the surface energy and is favored by intrinsic properties of PDMS Sylgard 184 for which several atomic force microscopy studies have shown that the roughness of a free PDMS surface is of the order of a few nanometers ${ }^{52,63,64}$. Moreover, the particles are between $10^{2}$ and $10^{4}$ times softer than the particles that usually constitute non-Brownian suspensions (mainly PS, PMMA or glass particles). As a consequence the force necessary to flatten a roughness is very small compared to the force induced by the flow in a typical rheometric experiment. The magnitude of the force required to flatten an asperity of radius of curvature $h_{r} \lesssim 10 \mathrm{~nm}$ can be estimated using Hertz theory: $F \approx 4 / 3 E^{*} h_{r}^{2}$ with $E^{*}=E /\left(1-v^{2}\right)$ and $v \approx 0.5$, which results in $F \lesssim 1 n N$ for the hardest PDMS particles and $F \approx 0.1 n N$ for the softest ones. These forces are much lower than those involved in the rheology experiments presented below where $F \approx 6 \pi a^{2} \sigma \approx 60 n N$ for $\sigma=1 P a$ that is the lower limit of the shear stresses we will explore. As a result, the roughness would not play any role and the interparticle contacts are expected to be soft. Moreover, PDMS is fully elastic over an extremely wide range of deformation and stress values ${ }^{49}$. From the mechanical properties of Sylgard 184 reported by Johnston et al. ${ }^{49}(E=2.05 M P a$, $v=0.5$ and $Y_{0}=6.25 \mathrm{MPa}$ at $T_{\text {curing }}=100^{\circ} \mathrm{C}$ ), the critical stress for plastic yield inception can be calculated from Eq. (6) with $h_{r}=a$ (since the contact is smooth) and a value of $\sigma_{c}$ as high as 
$10^{8} \mathrm{~Pa}$ is obtained! For the sake of comparison, Lobry et al. ${ }^{21}$ found $\sigma_{c} \approx 10 \mathrm{~Pa}$ for a suspension of $40 \mu \mathrm{m}$ diameter polystyrene beads (roughness of $\sim 100 \mathrm{~nm}$ ). This huge difference comes from the difference in Young modulus and yield strength values (respectively 1000 and 10 times lower for PDMS than for polystyrene) but, over all, from the difference in radius of curvature of the contact. In the case of rigid and rough particles, it is the radius of curvature of the asperities which intervenes while for PDMS particles that are soft and smooth, the radius of curvature to be taken into account to deduce the contact properties is the particle radius. As a consequence, in the shear stress range that will be explored in the present study $[1-150 \mathrm{~Pa}]$, the contact is fully elastic and described by JKR theory that relates the contact radius, $r_{c}$ to the applied load, $F$ :

$$
r_{c}=\left(\frac{3}{4} \frac{1-v^{2}}{E} a\right)^{1 / 3}\left(F+\frac{3}{2} \pi w_{S} a+\sqrt{3 \pi w_{S} a F+\left(\frac{3}{2} \pi w_{S} a\right)^{2}}\right)^{1 / 3}
$$

For applied loads much higher than $F_{a d h}$, this expression reduces to Hertz formula according to which the radius of the contact area varies as the normal load to the power $(1 / 3)$ :

$$
r_{c}=\left(\frac{3}{4} \frac{1-v^{2}}{E} a F\right)^{1 / 3}
$$

The friction coefficient is defined by the ratio of the normal force to the tangential force $\left(\mu=\frac{F_{t}}{F_{n}}\right)$ which is given by the product of frictional stress, $\Sigma_{f}$ and the contact area: $F_{t}=\Sigma_{f} \pi r_{c}^{2}$

As a result, in the limit $F>>F_{a d h}$, the friction coefficient is expected to decrease as $F^{-1 / 3}$ :

$$
\mu=\pi \Sigma_{f}\left(\frac{3}{4} \frac{1-v^{2}}{E} a\right)^{2 / 3} F^{-1 / 3}
$$

Furthermore, this expression shows that, at given normal load, the friction coefficient is proportional to the elastic modulus to the power of $(-2 / 3)$.

An order of magnitude of the frictional stress, $\Sigma_{f}$ for PDMS surfaces in air can be found in literature and is of the order of $0.2-0.3 \mathrm{MPa}^{65-67}$. In addition, it has been shown that friction is influenced by the physicochemical properties of the surrounding medium. In particular, Bogaerts et al. ${ }^{68}$ investigated the influence of wettability and surface roughness on the tribological properties of Sylgard 184 in the presence of a lubricant. In the boundary regime -where the lubricant is almost fully absent from the contact zone-, the authors showed that, in the presence of a nonwetting liquid, the friction coefficient is approximately the same as in dry contact while the friction coefficient decreases with decreasing contact angle. For a contact angle of about $35^{\circ}$, obtained with Sun Flower oil, they measured a boundary friction coefficient 3 times lower than the dry 
coefficient. Thus, since the wetting properties of PDMS by sun flower and Span 80 are nearly the same, $\Sigma_{f} \approx 0.1 \mathrm{MPa}$ can be taken as an order of magnitude for the frictional stress of the PDMS particles immersed in Span 80.

The frictional behavior of the glass beads is expected to be much different. Again, an order of magnitude of $\sigma_{c}$ can be calculated from the material properties of soda-lime glass ${ }^{69-71}: E \approx$ $70 \mathrm{GPa}, Y_{0} \approx 50 \mathrm{MPa}$ and $v \approx 0.22$ with $h_{r} \approx 5 \mathrm{~nm}$, which give $10^{-6} \mathrm{~Pa}$ for the estimation of $\sigma_{c}$. Thus, in the rheometric experiments presented below, the shear stress will always be much higher than $\sigma_{c}$. The friction coefficient is thus expected to be constant and no shear-thinning stemming from variable friction should be observed.

\section{Summary}

The particles that constitute the three suspensions that are studied here have very different mechanical properties with stiffness that varies over more than 4 decades and friction coefficient over almost one order of magnitude. By contrast, the adhesive properties of the two PDMS particle suspensions are essentially the same since, according to the JKR theory, the characteristic adhesive force does not vary with the elastic modulus of the particles ${ }^{62}$. The main characteristics of the suspensions are summarized in Table I.

\section{RHEOMETRIC EXPERIMENTS}

The measurements are carried out with a controlled-stress rheometer (Mars II, Thermofisher) equipped with a Pelletier temperature controller that sets the temperature within a precision of $\pm 0.1^{\circ} \mathrm{C}$. A parallel-plate geometry (radius $R=30 \mathrm{~mm}$, gap width $h=2.2 \mathrm{~mm}$ ) was employed in

order to minimize shear-induced particle migration ${ }^{75,76}$. Moreover, using a parallel-plate geometry makes it possible to work with large enough gaps so that particle layering in the wall region does not significantly affect the viscosity measurements ${ }^{47,77-79}$. Zarraga et al. ${ }^{80}$ showed that for a concentrated suspension $(\phi=0.5)$, viscosity approaches the continuum value as the gap width exceeds a value of about $40 \mathrm{a}$, a being the particle radius. Here the gap is set to $h=2.3 \mathrm{~mm}$ which corresponds to $h \gtrsim 40 a$ so that no confinement effect is expected.

The drawback of using a parallel plate geometry is the radial variation of the shear rate from 0 at the center to $\dot{\gamma}_{R}=\Omega R / h$ at the rim. To circumvent this difficulty, a correction is calculated 
TABLE I. caption

\begin{tabular}{cccc}
\hline \hline Particles & Soft PDMS & Stiff PDMS & Glass \\
\hline Suspending fluid & Span 80 & Span 80 & polytungstanate \\
& & & solution \\
$\eta_{0}(\mathrm{~Pa} . \mathrm{s})$ & 1.46 & 1.46 & 0.19 \\
$2 \mathrm{a}(\mu \mathrm{m})$ & $108 \pm 18$ & $115 \pm 13$ & $90 \pm 5$ \\
$\sigma_{\text {adh }}(\mathrm{Pa})$ & $\sim 3$ & $\sim 3$ & not evaluated \\
$\mathrm{E}(\mathrm{MPa})$ & $1.8 \pm 0.2$ & $15 \pm 2$ & $\sim 70.10^{3}$ \\
$\sigma_{c}(\mathrm{~Pa})$ & $\sim 10^{8}$ & $\sim 10^{8}$ & $\sim 10^{-6}$ \\
$\mu$ & $5.4 \sigma^{-1 / 3}$ & $1.3 \sigma^{-1 / 3}$ & $\sim 0.25^{\mathrm{a}}$ \\
volume fraction & $0.21-0.42$ & $0.33-0.525$ & $0.34-0.54$ \\
\hline \hline
\end{tabular}

a This value is taken from ${ }^{72}$ for contaminated particles in pure water but some authors report a decrease by a factor of up to 4 of the friction coefficient when silica particles are immersed in an electrolyte solution rather than in pure water $^{73,74}$

according to the Mooney-Rabinovitch formula:

$$
\sigma=\frac{\Gamma}{2 \pi R^{3}}\left(3+\frac{d \ln \Gamma}{d \ln \dot{\gamma}_{R}}\right)
$$

where $\Gamma$ is the torque applied by the rheometer.

The viscosity is then obtained :

$$
\eta=\frac{\sigma}{\dot{\gamma}_{R}}
$$

Viscosity is measured by imposing stress steps whose duration varies, depending on the intensity of the stress. Indeed, the steady viscosity of a suspension is only reached when its microstructure is at equilibrium, which requires that the shear deformation is of the order of several units ${ }^{14}$. Each measuring step is separated by a preshear at $\sigma=50 \mathrm{~Pa}$ for $20 \mathrm{~s}$ to prevent possible memory effects and to rehomogenize the suspension if necessary.

First, the effect of wall roughness on the viscosity measurement is tested on a suspension of soft PDMS particles $(E=1.8 \mathrm{MPa})$ at an intermediate concentration of 0.31 (as a reminder, the volume fraction of these particles is varied from 0.21 to 0.42 ). The rough surfaces are obtained by covering the nominally smooth surfaces of the lower and upper disks of the rheometer with a 


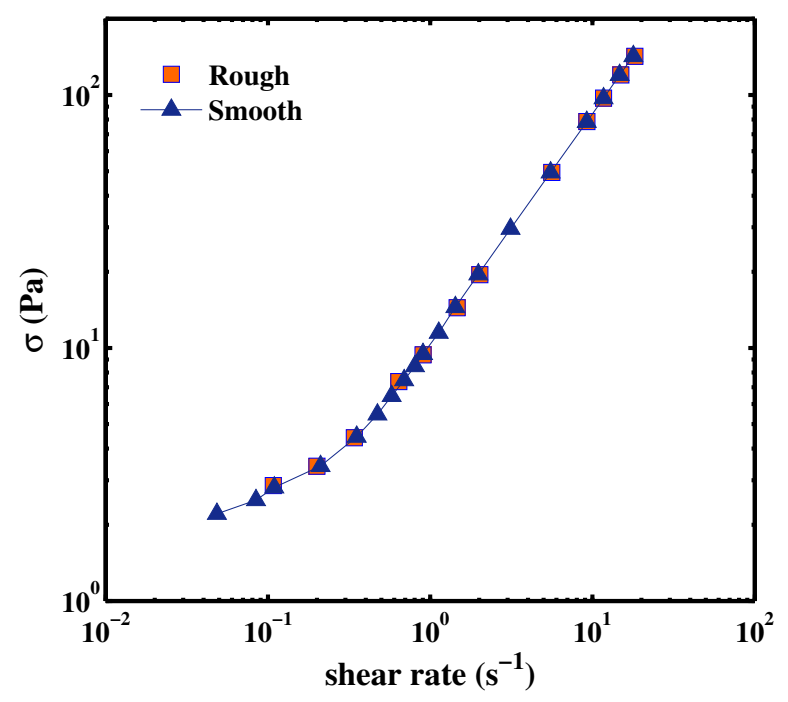

FIG. 6. Variation of shear stress as a function of shear rate measured either with smooth (triangles) or rough (squares) surfaces. The suspension is made of soft PDMS particles at a particle volume fraction of 0.31.

thin layer of PDMS (approximately $100 \mu \mathrm{m}$ in width) which is seeded before curing with $80 \mu \mathrm{m}$ diameter glass. Fig. 6 displays the variation of shear stress as a function of shear rate measured either with smooth or rough surfaces. Since no difference is observed, in the following, unless specified otherwise, viscosity will be measured with smooth surfaces whose use ensures a better parallelism of the rheometer disks and a more accurate evaluation of the gap width.

Figure 7 shows the shear stress-shear rate curves obtained for the three systems at different particle volume fractions. Whereas the glass bead suspension is essentially Newtonian (only a slight deviation of the linear relation between $\sigma$ and $\dot{\gamma}$ is observed for the highest concentrations), the PDMS particle suspensions exhibit shear-thinning behaviors that are more pronounced the higher the particle concentration and the smaller the Young modulus of the particles. Moreover, we notice that for a given volume fraction, the viscosity of the suspension of soft PDMS particles is greater than that of the suspension of stiff PDMS particles, which is itself somewhat greater than the viscosity of the glass bead suspension. 

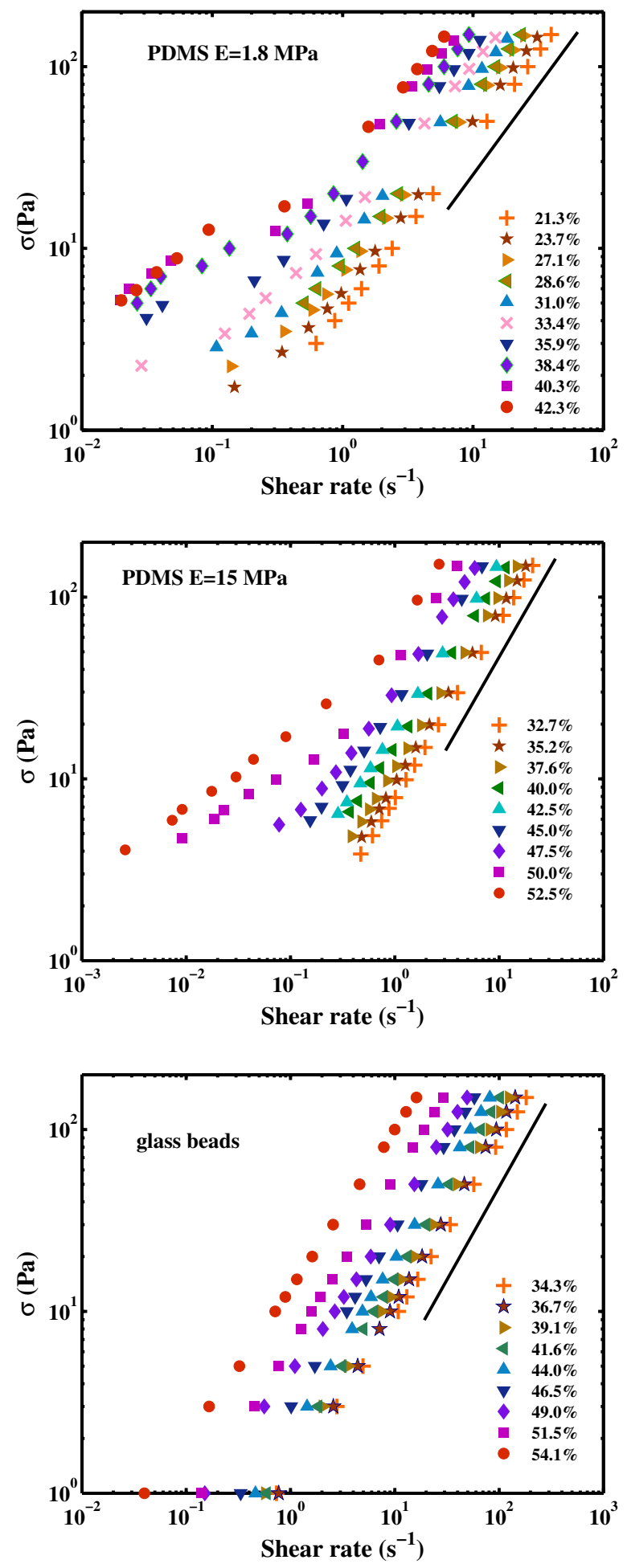

FIG. 7. Shear stress versus shear rate for the three kinds of suspensions at several particle volume fractions. It is observed that the glass bead suspensions exhibit a Newtonian behavior, whatever the volume fraction. On the contrary, suspensions of PDMS particles show a non-Newtonian behavior which is more pronounced the higher the particle volume fraction and the lower the stiffness of PDMS. 


\section{RESULTS AND DISCUSSION}

\section{A. Jamming fraction as a function of the shear stress}

In order to provide a synthetic presentation of the large number of results shown in Fig. 7 and to be able to compare quantitatively the behaviors observed for the three types of suspensions, we will follow the approach of several authors that consists in considering that the jamming fraction, $\phi_{m}$ is dependent on the stress magnitude ${ }^{13,20-22,32,35,41}$. The variation of viscosity with particle volume fraction and shear stress is depicted using a modified Maron-Pierce law ${ }^{81}$ :

$$
\eta_{S}=\frac{\eta}{\eta_{0}}=\frac{\alpha(\sigma)}{\left(1-\frac{\phi}{\phi_{m}(\sigma)}\right)^{2}}
$$

where $\alpha$ is a fitting parameter of the order of 1 without any clear physical meaning. In the original Maron-Pierce relation $\alpha=1$ while almost all the numerical or experimental studies that use Eq. (19) to represent the viscosity as a function of the volume fraction report values of $\alpha$ that deviate from $1^{17,21}$.

Fig. 8 shows the variation of $1 / \eta_{S}^{0.5}$ as a function of particle volume fraction for a few different stress magnitude. For the three types of suspensions a linear behavior with $\phi$ is observed. For the glass bead suspensions, almost no variation of the jamming fraction ( $\phi$-axis intercept) with stress is observed, which is consistent with the Newtonian behavior shown in Fig. 7. In contrast, for both PDMS particle suspensions, the jamming volume fraction noticeably decreases with decreasing shear stress.

Fig. 9 displays the variation of $\alpha$ with $\sigma$ for the three suspensions. As expected, $\alpha$ is of the order of unity. For the glass bead suspension, $\alpha$ is remarkably constant while it somewhat increases with increasing $\sigma$ for both suspensions of PDMS particles. This moderate increase of $\alpha$ has already been observed in both experimental ${ }^{35}$ and numerical ${ }^{21}$ studies. $^{2}$

The variation of $\phi_{m}$ with $\sigma$ is much more interesting and meaningful; it is shown in Fig 10 . First, we note the low values of the jamming volume fraction obtained for PDMS suspensions when the shear stress decreases towards zero. These values, typically ranging from 0.4 to 0.5 , are of the same order of magnitude as the values of random loose packing ${ }^{82}$ or of jamming fraction ${ }^{83}$ in cohesive granular materials. Second, the variation of $\phi_{m}$ with $\sigma$ is very significant for PDMS particle suspensions and is greater for the soft PDMS particle suspension than for the stiff PDMS particle suspension while, as already mentioned, the jamming fraction of the glass particle sus- 

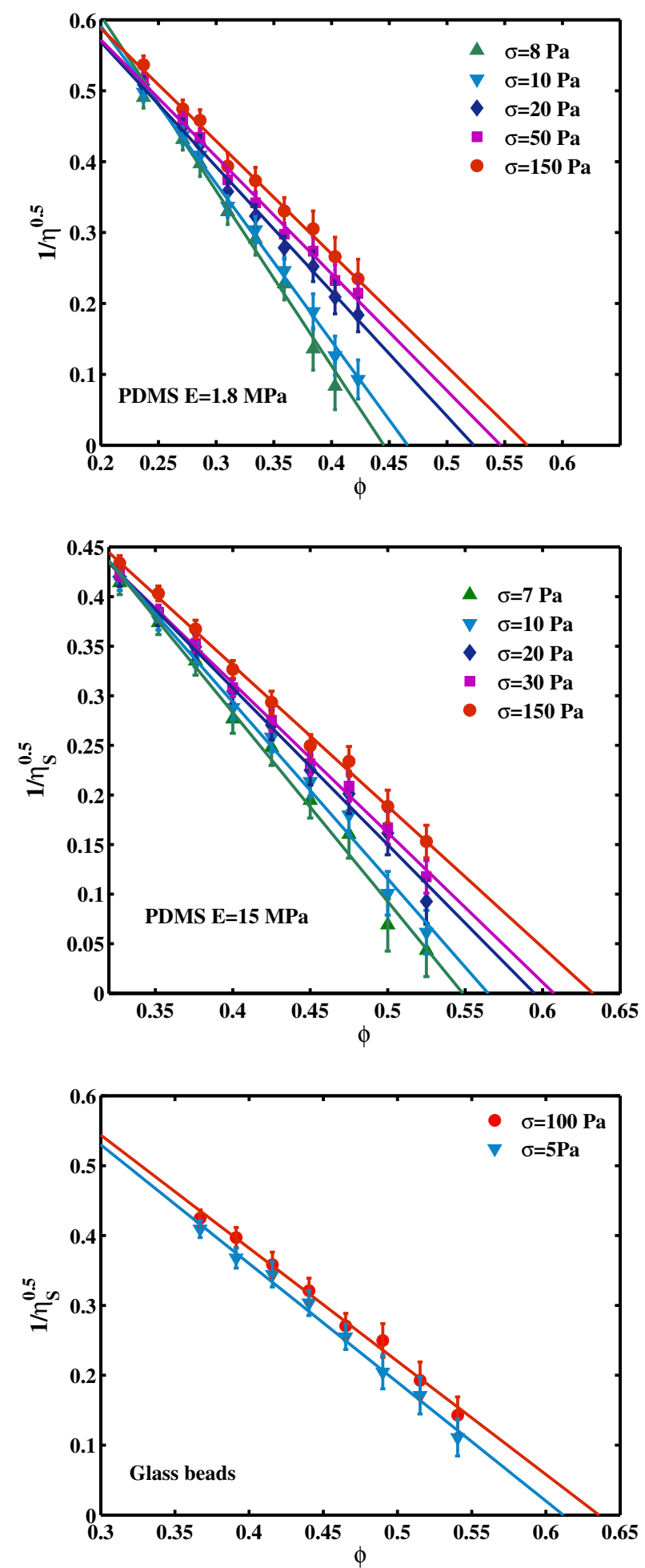

FIG. 8. Inverse of the square root of the reduced viscosity versus particle volume fraction for several shear stress values. 


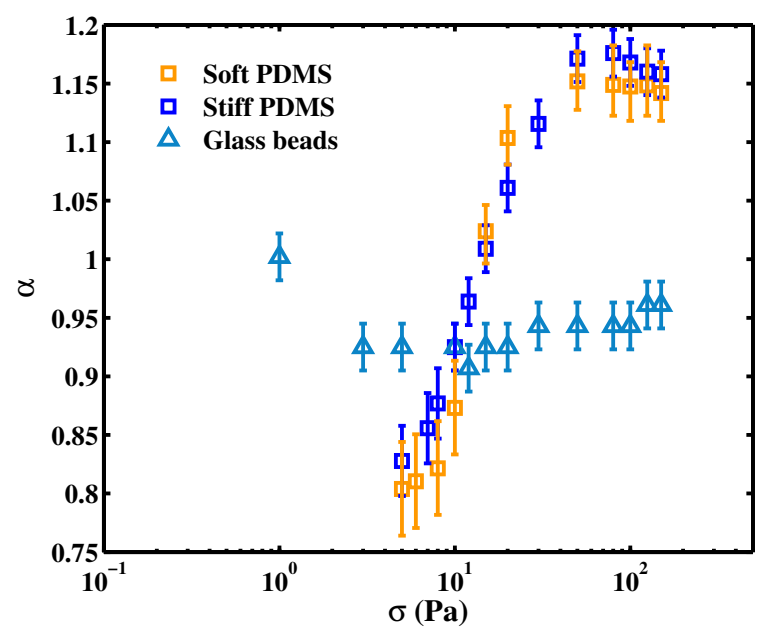

FIG. 9. $\alpha$ parameter (Eq. (19)) versus shear stress.

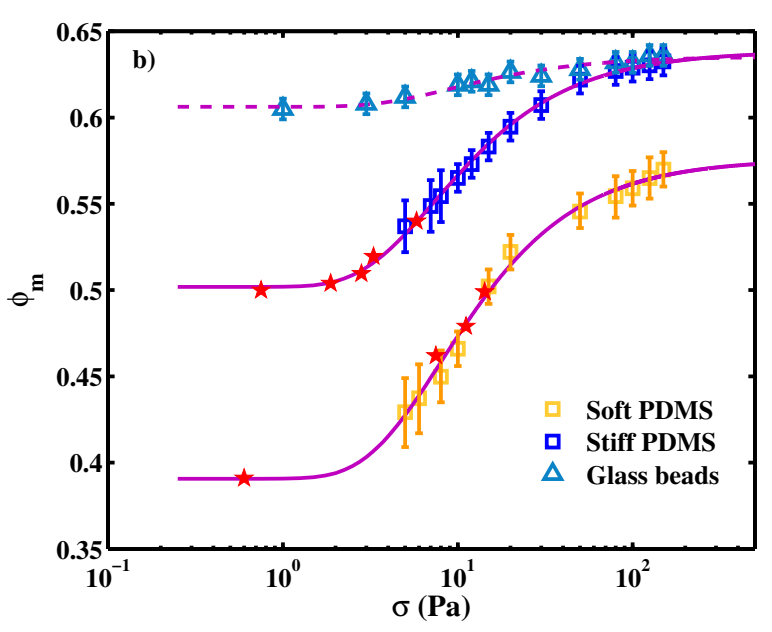

FIG. 10. Jamming volume fraction versus shear stress. Hollow symbols correspond to the determination of $\phi_{m}$ from the variation of $1 / \eta_{S}^{1 / 2}$ as a function of $\phi$ (see Eq. (19)). Red stars represent the variation of the yield stress, $\sigma_{S}$, with particle volume fraction (see Sec. V B for the determination of the yield stress). The solid lines are obtained by fitting the whole set of experimental results $\left(\phi_{m}(\sigma)\right.$ and $\left.\sigma_{S}(\phi)\right)$ by the model proposed by Richards et al. ${ }^{12}$. The fitting parameters are given in Tab. II. The dashed line has only been added to guide eye.

pension barely varies with shear stress, which is consistent with the quasi-Newtonian behavior displayed in Fig 7. We also note that the differences recorded in the values of $\phi_{m}$ for the three types of suspensions are much more marked at low stresses than at high stresses. Indeed, for $\sigma=150 P a$, the glass particle suspension and the stiff PDMS particle suspension have approxi- 
mately the same jamming fraction $(\approx 0.63)$. The jamming fraction of the soft PDMS suspension is smaller $(\approx 0.57)$ but the experimental data of Fig. 10 show that $\phi_{m}$ has not reached a constant value and would probably continue to increase if higher stresses were applied.

\section{B. Yield stress}

The very important variation of $\phi_{m}$ with $\sigma$ should result in the emergence of a yield stress in suspensions whose particle volume fraction is comprised between the minimum jamming fraction, $\phi_{a l p}$, and the maximum one, $\phi_{\mu}$. Indeed, in this concentration range, the suspension will only flow if the stress magnitude is large enough for the jamming fraction to be larger than its volume fraction. We wanted to test this idea and for this purpose we made new suspensions of both kinds of PDMS particles with volume fractions between $\phi_{a l p}$ and $\phi_{\mu}$ and measured the yield stress, $\sigma_{S}$. To measure as precisely as possible the yield stress, a sequence of steps of decreasing shear stresses is applied to the suspension. When changing from a given stress to a smaller stress, the elastic particles relax part of their contact forces and induce a reverse of the flow of the suspension. After a lapse of time, if the shear stress is larger than the yield stress, the suspension flows again in the direction of the applied stress. On the contrary, if the applied stress is lower than the yield stress, the suspension stops, once the contact forces between particles have been relaxed and never flows again. Thus, to measure $\sigma_{S}$, we record the minimum value of the shear stress which induces forward flow. An example of such a test is given in Fig.11 and the recorded values of $\sigma_{S}$ for various particle volume fractions are represented in Fig 10 by the red stars.

An important feature on this figure is the very good correspondence between the curves $\sigma_{S}(\phi)$ ans $\phi_{m}(\sigma)$ which confirms the suggestion we made that a variation of $\phi_{m}$ with $\sigma$ should give rise to the emergence of a yield stress.

\section{Interpretation of the results}

The very low values of $\phi_{m}$ (as low as 0.43 for the softer PDMS particles) recorded at small applied shear stresses show that the framework of purely frictional suspensions is not appropriate to describe our results. Indeed, for frictional suspensions, the jamming volume fraction can only vary in a range typically between $\phi^{\infty} \approx 0.55$ (for $\mu \rightarrow \infty$ ) and $\phi^{0} \approx 0.65-0.7$ (for $\left.\mu \rightarrow 0\right)^{16,18,20,21}$. Recent papers ${ }^{12,27}$ focused on the role of adhesive forces in rheology of non-Brownian suspensions 


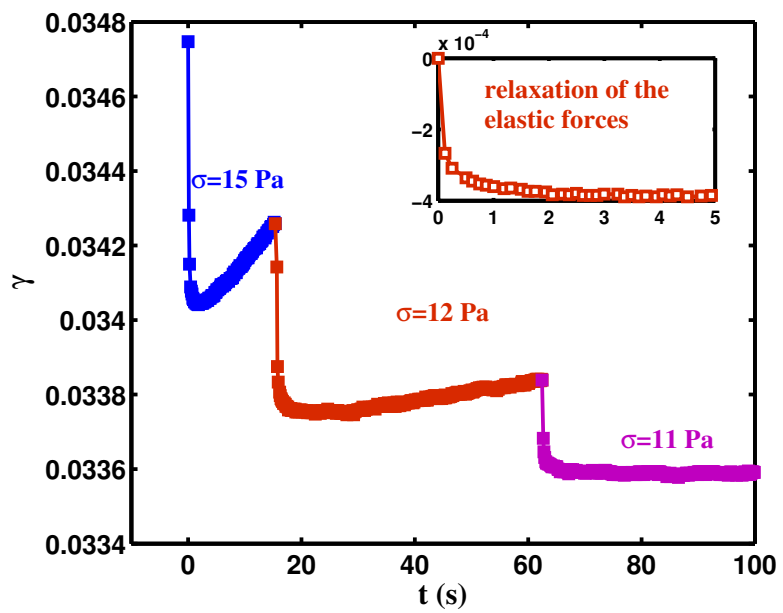

FIG. 11. Shear strain versus time for different shear stress values. When the stress is switched from a given value to a lower one, part of the interparticle elastic forces relax, which leads to a strain recovery. Afterwards, if the applied stress is greater than the yield stress, the strain increases again. Otherwise, the deformation levels off. This test example was performed with a suspension of soft PDMS particles at a volume fraction of 0.479 . The curves indicate that the yield stress is between 11 and $12 \mathrm{~Pa}$. Smaller stress intervals between these two values allow us to evaluate with accuracy the value of the yield stress at $11.2 \pm 0.1 P a$

and showed that adhesion is responsible for shear-thinning and may give rise to jamming fraction values as low as 0.3. In the phenomenological model proposed by Richards et al. ${ }^{12}$ (see Sec II), $\phi_{a l p}$ corresponds to the jamming fraction at $\sigma \rightarrow 0$ for a suspension where all contacts are adhesive and $\phi_{\mu}$ is the jamming volume fraction for a non-adhesive frictional suspension whose value only depends on the friction coefficient between particles ${ }^{16,18,20,21,40}$. The suspension transits from the adhesive behavior to the frictional one for a characteristic stress $\sigma_{a}$ associated with the force that is necessary to break an adhesive contact. In the following, we will analyze our data using this constraint-based rheology model and, to limit the number of parameters, we will set the value of the parameter $\kappa$ that appears in Eq. (4) to 1. The solid lines in Fig. 10 show the results of the fitting of the experimental data with the model of Richards et al. Note that all the values $\phi_{m}(\sigma)$, as well as $\sigma_{S}(\phi)$, are used to perform the fits. Table II displays the fitting parameters for both kinds of PDMS particle suspensions.

The characteristic adhesive stress is almost the same for both suspensions. This finding is 
TABLE II. caption

\begin{tabular}{cccc}
\hline \hline Suspensions & $\phi_{a l p}$ & $\phi_{\mu}$ & $\sigma_{a}$ \\
\hline PDMS Sylgard 184 $(E=1.8 \mathrm{MPa})$ & $0.391 \pm 0.005$ & $0.576 \pm 0.008$ & $8.1 \pm 1.0$ \\
PDMS Sylgard 184 + Toluene $(E=15 \mathrm{MPa})$ & $0.502 \pm 0.005$ & $0.639 \pm 0.007$ & $7.5 \pm 1.5$ \\
\hline \hline
\end{tabular}

TABLE III. Comparison of the $\phi_{m}$ values obtained either experimentally or predicted by Eq. (16) together with constitutive laws proposed by Singh et al. ${ }^{17}$ and by Lobry et al. ${ }^{21}$

\begin{tabular}{cccc}
\hline \hline & Glass beads & Stiff PDMS & Soft PDMS \\
$\mu(\sigma=150 P a)$ & 0.25 & 0.25 & 1.0 \\
$\phi_{m}$ experimental & 0.636 & 0.633 & 0.570 \\
$\phi_{m}$ (Singh et al. $\left.{ }^{17}\right)$ & 0.614 & 0.614 & 0.580 \\
$\phi_{m}$ (Lobry et al. $\left.^{21}\right)$ & 0.631 & 0.631 & 0.569 \\
\hline \hline
\end{tabular}

consistent with JKR theory that predicts that the force necessary to break an adhesive contact between two spheres does not depend upon the elastic modulus of the materials (see Eq.(13)). Furthermore, the characteristic adhesion force can be evaluated from Eqs. (5) and (13): $\sigma_{a d h} \approx \frac{w_{S}}{8 a}$. Taking $w_{S} \sim 1.25 \mathrm{~mJ} / \mathrm{m}^{2}$ (see Sec. III C 3), we obtain $\sigma_{a d h} \sim 3 P a$ which is of the same order of magnitude as the value of $\sigma_{a}$ deduced from rheometric experiments together with the model of $\operatorname{Richards}^{12}\left(\sigma_{a} \approx 8 P a\right)$.

For stresses well above $\sigma_{a}$, the suspensions is expected to behave like a "standard" frictional suspension with a jamming fraction controlled by the magnitude of the inter-particle friction coefficient, $\mu$. As explained in Sec. II, since the contact is smooth and the PDMS is an almost ideally elastic material, $\mu$ is expected to decrease with the normal force to the power $(-1 / 3)$ (see Eq. (16)). Eq. (5), together with Eq. (16) and $\Sigma_{f} \approx 0.1 M P a$, give $\mu \approx 1$ for the softer particles and $\mu \approx 0.25$ for the stiffer ones at a shear stress $\sigma=150 \mathrm{~Pa}$. For the latter, the friction coefficient is of the same order as that of glass beads. It is thus consistent that, at high shear stresses, the jamming fraction is almost the same for both kinds of suspensions. In addition, the $\phi_{m}$ values obtained for the three systems can be compared to the results of numerical simulations from Singh et al. ${ }^{17}$ or from Lobry et al. ${ }^{21}$ who obtained values of $\phi_{m}$ that are very close to that we have measured (see Tab. III).

The values of $\phi_{a l p}$ are somewhat more difficult to interpret. On the one hand, according to JKR 
theory, the characteristic adhesion force which is the force necessary to separate two spheres does not depend on the sphere stiffness, which, as stressed before, is consistent with the observation that the characteristic stress deduced from rheometric measurements is the same for both kinds of PDMS particle suspensions. But, on the other hand, as long as the particles are in contact, the contact area varies with the stiffness. Eq. (14) shows that, at zero applied external force, the radius of the contact area varies as $E^{-1 / 3}$. Thus, when the sphere stiffness decreases, the attractive adhesion force between the particles, which is proportional to the contact area, increases as $E^{-2 / 3}$. This variation of contact area with particle stiffness provides an explanation for the smaller value of $\phi_{a l p}$ measured for soft PDMS spheres than for stiff PDMS spheres. Note that, in the absence of adhesion, the difference in stiffness should have led to a slightly larger value of $\phi_{a l p}$ for the suspension of softer spheres since they are more deformable and can arrange in a more compact structure. This has been shown by Martin \& Bordia ${ }^{84}$ who studied the influence of stiffness, adhesion and friction on random close packing fraction of spherical particles. Other authors ${ }^{37-39}$ have also studied the role of adhesion and friction on the maximum packing fraction. In these numerical studies, particles settle under gravity and the packing fraction is characterized as a function of the friction coefficient and of the ratio of the adhesion force to the buoyant force. The main result of these studies is that the packing fraction decreases with the force ratio up to a limit value that depends on the value of the friction coefficient. Liu et al. ${ }^{39}$ showed that $\phi_{\text {alp }}$ typically decreases from 0.5 to 0.2 when $\mu$ increases from 0 to 1 . These orders of magnitude are consistent with the values of $\phi_{a l p}$ that we measured. However, according to Liu et al. the strong decay of $\phi_{a l p}$ occurs for friction coefficients of the order of $10^{-3}-10^{-2}$ while, for higher values of $\mu, \phi_{\text {alp }}$ hardly decreases. Therefore, since the values of the friction coefficient of both kinds of PDMS particles are much larger than the typical values at which the variation of $\phi_{a l p}$ is significant, sliding friction alone could not explain either the very low values of $\phi_{a l p}$ or the difference in $\phi_{a l p}$ values measured for each of the two suspensions. Yang et al. ${ }^{38}$ showed the same kind of variation in $\phi_{a l p}$ with $\mu$ but also that the rolling friction coefficient, $\mu_{r}$, has a significant influence on $\phi_{a l p}$ when it varies between 0.001 and 0.1 .

The rolling friction force involved in a JKR contact between two spherical particles has been derived by Dominik \& Tielens ${ }^{85}$ :

$$
F_{r}=3 \pi w_{S} \xi^{\text {yield }}
$$

where $\xi^{\text {yield }}$ is a critical rolling displacement for the onset of rolling. We thus obtain the rolling 
friction coefficient:

$$
\mu_{r}=\frac{F_{r}}{F_{a d h}}=4 \frac{\xi^{\text {yield }}}{a}
$$

Krijt et al. ${ }^{86}$ proposed a relation between $\xi$ and the amount of adhesion hysteresis, $\Delta w_{S}$, and the radius of the contact area by considering the changes of contact area and pressure distribution at the front and rear sides of the contact region:

$$
\xi^{\text {yield }}=\frac{r_{c}}{12} \frac{\Delta w_{S}}{w_{S}}
$$

where $r_{c} \approx r_{e q}=\left(\frac{9 \pi w_{S}\left(1-v^{2}\right) a^{2}}{4 E}\right)^{1 / 3}$ is the radius of the contact area when no external force is applied (see Eq. (14) with $\mathrm{F}=0$ ). Here, we obtain $r_{c} \approx 2 \mu \mathrm{m}$ for the softer PDMS particles and $r_{c} \approx 1.1 \mu \mathrm{m}$ for the stiffer ones. Several studies have measured the adhesion hysteresis of PDMS in air. The value of the adhesion hysteresis depends on many parameters, including free chain extraction (or not) ${ }^{87,88}$, mixing ratio ${ }^{54,88}$, molecular weight ${ }^{89,90}$, formation of hydrogen bonds across the interface ${ }^{87,89,90} \ldots$ Nevertheless, the compilation of the results of the literature shows that for extracted Sylgard $184 \Delta w_{S} / w_{s} \approx 1$. This order of magnitude together with the contact radius value that is much larger than for usually much stiffer particles (PMMA, PS, silica...) lead to a quite unusual large value of the critical rolling displacement which is of several hundred nanometers, resulting in rolling friction coefficients of about 0.012 for the softer particles and 0.007 for the stiffer ones.

The effect of rolling friction on suspension rheology has been seldom studied, probably because, for stiffer spherical particle suspensions, friction is dominated by sliding friction. However, a recent paper addressed, through numerical simulations, the influence of rolling friction on the DST transition and more generally on the rheology of suspensions in the thickened frictional regime $^{91}$. The authors show that rolling friction results in a decrease of the jamming fraction in a range qualitatively comparable to what we observe here. For $\mu=10$ (here for the softest particles $\mu \approx 12$ is obtained by introducing the value of the adhesion force in Eq. (16), they obtain a jamming fraction of 0.4 for $\mu_{r} \approx 1$, a value 50 times larger than the one we estimated from the adhesion hysteresis. This discrepancy probably means that adhesion cannot be taken into account only through rolling friction. In other words, adhesion, in addition to constraining the tangential motion of particles, prevents particles from separating and this effect, which is not accounted for by rolling friction, is likely to have an important impact on the suspension rheology. However, this negative result must be taken with caution because it is not excluded that we have underestimated 
the rolling friction coefficient. On the one hand, the theory that links $\mu_{r}$ to the adhesion hysteresis has not yet received much experimental confirmation. On the other hand, the order of magnitude that we used for $\Delta w_{S} / w_{S}$ is derived from the literature which shows that the value of this hysteresis depends very much on the precise characteristics of the PDMS studied. In order to make a final decision on the possibility to account for the effect of adhesion on rheology by introducing a rolling friction, we believe that it would be necessary, even though it is quite challenging, to measure directly the rolling friction coefficient of the particles.

\section{CONCLUDING REMARKS}

We designed a simple millifluidic device to manufacture PDMS particles with a diameter of $\approx 100 \mu \mathrm{m}$ and with two different elastic moduli: 1.8 MPa for the softer PDMS particles and $15 \mathrm{MPa}$ for the stiffer ones. Due to the low values of capillary number investigated (lower than

$10^{-4}$ ), particles are not expected to be significantly deformed by the flow. However, the impact of particle rigidity on suspension rheology is evidenced. The rheological behavior of suspensions of stiff and soft PDMS particles dispersed in Span 80 is compared to the rheological behavior of suspensions of glass beads immersed in a Newtonian liquid. The glass suspensions exhibit a quasi Newtonian behavior while the PDMS particle suspensions display a marked shear-thinning behavior. As the modulus decreases, the viscosity increases, especially at low shear stress, and the shear-thinning behavior becomes more pronounced. In the three cases, shear-thinning is quantified by the variation of the jamming fraction with shear stress. The experimental results are interpreted on the basis of the constraint based model proposed by Guy et al. ${ }^{36}$ and Richards et al. ${ }^{12}$ which involves three parameters: the characteristic adhesion stress, $\sigma_{a}$, the jamming volume fraction in the adhesive regime $\left(\sigma<<\sigma_{a}\right), \phi_{a l p}$, and the jamming volume fraction in the frictional regime $\left(\sigma>>\sigma_{a}\right), \phi_{\mu}$

The adhesion stress is found to be equal for both PDMS particle suspensions, in agreement with the JKR theory that predicts that the pull-off force required to separate two adhesive spheres is independent of the sphere stiffness. Moreover the magnitude of the adhesion stress is fully compatible with the magnitude of the adhesion energy, estimated from the physicochemical properties of PDMS and span 80. In the future, it would be interesting to change the suspending liquid to vary the adhesion energy and observe the effects on the rheology and more particularly on the value of the characteristic adhesion stress. 
The values of $\phi_{\mu}$ for both kinds of PDMS particle suspensions are consistent with the evaluated values of the friction coefficient together with the results of numerical simulations from Singh $e t$ $a l .{ }^{17}$ or Lobry et al. ${ }^{21}$. The value of the jamming fraction measured for the glass bead suspension is almost independent of $\sigma$ since the suspensions exhibit a quasi Newtonian behavior, and is also in agreement with the numerical simulation results mentioned above. It should also be stressed that the lack of shear-thinning in the glass beads suspensions is consistent with the predictions of the model proposed by Lobry et al. ${ }^{21}$ which shows that non adhesive frictional suspensions are expected to display shear-thinning behavior in a range of shear stress values lower or the order of magnitude of the critical shear stress that corresponds the plastification of the interparticle contacts where particle contact is expected to be plastic.

The values found for $\phi_{a l p}$ are within the expected range for adhesive suspensions ${ }^{12,13,27}$. The comparison of these values $\left(\phi_{a l p}=0.39\right.$ and 0.50 for the soft and stiff PDMS particle suspensions respectively) with the values deduced from numerical simulations ${ }^{91}$ that take into account both sliding and rolling friction, suggests that it is unlikely that the effect of adhesion can be described by only a rolling constraint of the particles.

The significant variation of $\phi_{m}$ (from $\phi_{a l p}$ to $\phi_{\mu}$ ) with shear stress leads to the emergence of a yield stress, $\sigma_{S}$, for suspensions with volume fractions between $\phi_{a l p}$ and $\phi_{\mu}$. We showed that, in agreement with the findings of Richards et al. ${ }^{12}$, the relations $\phi_{m}(\sigma)$ and $\sigma_{S}(\phi)$ are closely equivalent.

\section{ACKNOWLEDGMENTS}

We acknowledge Céline Cohen for her advice on the handling of PDMS, Caroline Fradin for the contact and interfacial tension measurements and Sandra Bosio and Christophe Pitiot for their help in designing and carrying out the experimental setups.

\section{REFERENCES}

${ }^{1}$ I. M. Krieger and T. J. Dougherty, “A mechanism for non-newtonian flow in suspensions of rigid spheres," Transactions of the Society of Rheology 3, 137-152 (1959).

${ }^{2}$ D. Quemada, "Rheological modelling of complex fluids. i. the concept of effective volume fraction revisited," The European Physical Journal Applied Physics 1, 119-127 (1998). 
${ }^{3}$ R. G. Owens, "A new microstructure-based constitutive model for human blood," Journal of non-newtonian fluid mechanics 140, 57-70 (2006).

${ }^{4}$ G. Barthelmes, S. E. Pratsinis, and H. Buggisch, "Particle size distributions and viscosity of suspensions undergoing shear-induced coagulation and fragmentation," Chemical Engineering Science 58, 2893-2902 (2003).

${ }^{5}$ R. Mondragon, J. E. Julia, A. Barba, and J. C. Jarque, "Characterization of silica-water nanofluids dispersed with an ultrasound probe: A study of their physical properties and stability," Powder Technology 224, 138-146 (2012).

${ }^{6}$ A. Acrivos, X. Fan, and R. Mauri, "On the measurement of the relative viscosity of suspensions," Journal of Rheology 38, 1285-1296 (1994).

${ }^{7}$ A. Vázquez-Quesada, R. I. Tanner, and M. Ellero, "Shear thinning of noncolloidal suspensions," Physical review letters 117, 108001 (2016).

${ }^{8}$ A. Vázquez-Quesada, A. Mahmud, S. Dai, M. Ellero, and R. I. Tanner, "Investigating the causes of shear-thinning in non-colloidal suspensions: Experiments and simulations," Journal of NonNewtonian Fluid Mechanics 248, 1-7 (2017).

${ }^{9}$ R. I. Tanner and S. Dai, “Particle roughness and rheology in noncolloidal suspensions,” Journal of Rheology 60, 809-818 (2016).

${ }^{10}$ Y. Lin, Y. Wang, H. Qin, D. Pan, and J. Chen, "Surface roughness effect on the shear thinning of non-colloidal suspensions," Physics of Fluids 33, 043104 (2021).

${ }^{11}$ M. Kroupa, M. Soos, and J. Kosek, "Slip on a particle surface as the possible origin of shear thinning in non-brownian suspensions," Physical Chemistry Chemical Physics 19, 5979-5984 (2017).

${ }^{12}$ J. A. Richards, B. M. Guy, E. Blanco, M. Hermes, G. Poy, and W. C. Poon, "The role of friction in the yielding of adhesive non-brownian suspensions," Journal of Rheology 64, 405-412 (2020).

${ }^{13}$ J. Z. Zhou, P. H. Uhlherr, and F. T. Luo, "Yield stress and maximum packing fraction of concentrated suspensions," Rheologica acta 34, 544-561 (1995).

${ }^{14}$ F. Blanc, F. Peters, and E. Lemaire, "Experimental signature of the pair trajectories of rough spheres in the shear-induced microstructure in noncolloidal suspensions," Physical review letters 107, 208302 (2011).

${ }^{15}$ P. Pham, B. Metzger, and J. E. Butler, "Particle dispersion in sheared suspensions: Crucial role of solid-solid contacts," Physics of Fluids 27, 051701 (2015).

${ }^{16}$ S. Gallier, E. Lemaire, F. Peters, and L. Lobry, "Rheology of sheared suspensions of rough 
frictional particles," Journal of Fluid Mechanics 757, 514-549 (2014).

${ }^{17}$ A. Singh, R. Mari, M. M. Denn, and J. F. Morris, “A constitutive model for simple shear of dense frictional suspensions," Journal of Rheology 62, 457-468 (2018).

${ }^{18}$ W. Chèvremont, B. Chareyre, and H. Bodiguel, "Quantitative study of the rheology of frictional suspensions: Influence of friction coefficient in a large range of viscous numbers," Physical Review Fluids 4, 064302 (2019).

${ }^{19}$ R. Seto, R. Mari, J. F. Morris, and M. M. Denn, "Discontinuous shear thickening of frictional hard-sphere suspensions," Physical review letters 111, 218301 (2013).

${ }^{20}$ R. Mari, R. Seto, J. F. Morris, and M. M. Denn, "Shear thickening, frictionless and frictional rheologies in non-brownian suspensions," Journal of Rheology 58, 1693-1724 (2014).

${ }^{21}$ L. Lobry, E. Lemaire, F. Blanc, S. Gallier, and F. Peters, "Shear thinning in non-brownian suspensions explained by variable friction between particles," Journal of Fluid Mechanics 860, 682-710 (2019).

${ }^{22}$ R. V. More and A. M. Ardekani, "Effect of roughness on the rheology of concentrated nonbrownian suspensions: A numerical study," Journal of Rheology 64, 67-80 (2020).

${ }^{23}$ R. Seto and G. G. Giusteri, "Normal stress differences in dense suspensions," Journal of Fluid Mechanics 857, 200-215 (2018).

${ }^{24}$ P. Pham, J. E. Butler, and B. Metzger, "Origin of critical strain amplitude in periodically sheared suspensions," Physical Review Fluids 1, 022201 (2016).

${ }^{25}$ J. Archard, "Elastic deformation and the laws of friction," Proceedings of the Royal Society of London. Series A. Mathematical and Physical Sciences 243, 190-205 (1957).

${ }^{26}$ V. Brizmer, Y. Kligerman, and I. Etsion, "Elastic-plastic spherical contact under combined normal and tangential loading in full stick," Tribology Letters 25, 61-70 (2007).

${ }^{27}$ A. Papadopoulou, J. J. Gillissen, H. J. Wilson, M. K. Tiwari, and S. Balabani, "On the shear thinning of non-brownian suspensions: friction or adhesion?" Journal of Non-Newtonian Fluid Mechanics , 104298 (2020).

${ }^{28}$ M. M. Villone and P. L. Maffettone, "Dynamics, rheology, and applications of elastic deformable particle suspensions: a review," Rheologica Acta 58, 109-130 (2019).

${ }^{29}$ S. Adams, W. Frith, and J. Stokes, "Influence of particle modulus on the rheological properties of agar microgel suspensions," Journal of Rheology 48, 1195-1213 (2004).

${ }^{30}$ H. M. Shewan and J. R. Stokes, "Viscosity of soft spherical micro-hydrogel suspensions," Journal of colloid and interface science 442, 75-81 (2015). 
${ }^{31}$ P. Snabre and P. Mills, "Ii. rheology of weakly flocculated suspensions of viscoelastic particles," Journal de Physique III 6, 1835-1855 (1996).

${ }^{32}$ M. Wyart and M. Cates, "Discontinuous shear thickening without inertia in dense non-brownian suspensions," Physical review letters 112, 098302 (2014).

${ }^{33}$ J. F. Morris, "Lubricated-to-frictional shear thickening scenario in dense suspensions," Physical Review Fluids 3, 110508 (2018).

${ }^{34}$ D. Quemada, "Rheology of concentrated disperse systems ii. a model for non-newtonian shear viscosity in steady flows," Rheologica Acta 17, 632-642 (1978).

${ }^{35}$ F. Blanc, E. d'Ambrosio, L. Lobry, F. Peters, and E. Lemaire, "Universal scaling law in frictional non-brownian suspensions," Physical Review Fluids 3, 114303 (2018).

${ }^{36}$ B. Guy, J. Richards, D. Hodgson, E. Blanco, and W. Poon, "Constraint-based approach to granular dispersion rheology," Physical review letters 121, 128001 (2018).

${ }^{37}$ R. Yang, R. Zou, and A. Yu, "Computer simulation of the packing of fine particles," Physical review E 62, 3900 (2000).

${ }^{38}$ R. Yang, R. Zou, and A. Yu, "Effect of material properties on the packing of fine particles," Journal of applied physics 94, 3025-3034 (2003).

${ }^{39}$ W. Liu, Y. Jin, S. Chen, H. A. Makse, and S. Li, "Equation of state for random sphere packings with arbitrary adhesion and friction," Soft Matter 13, 421-427 (2017).

${ }^{40}$ F. Peters, G. Ghigliotti, S. Gallier, F. Blanc, E. Lemaire, and L. Lobry, "Rheology of nonbrownian suspensions of rough frictional particles under shear reversal: A numerical study," Journal of rheology 60, 715-732 (2016).

${ }^{41}$ G. Chatté, J. Comtet, A. Niguès, L. Bocquet, A. Siria, G. Ducouret, F. Lequeux, N. Lenoir, G. Ovarlez, and A. Colin, "Shear thinning in non-brownian suspensions," Soft matter 14, 879893 (2018).

${ }^{42}$ R. I. Tanner, C. Ness, A. Mahmud, S. Dai, and J. Moon, “A bootstrap mechanism for noncolloidal suspension viscosity," Rheologica Acta 57, 635-643 (2018).

${ }^{43}$ J. Carneiro, J. Campos, and J. Miranda, "Pdms microparticles produced in pdms microchannels under the jetting regime for optimal optical suspensions," Colloids and Surfaces A: Physicochemical and Engineering Aspects 580, 123737 (2019).

${ }^{44} \mathrm{~S}$. Tomotika, "On the instability of a cylindrical thread of a viscous liquid surrounded by another viscous fluid," Proceedings of the Royal Society of London. Series A-Mathematical and Physical Sciences 150, 322-337 (1935). 
${ }^{45}$ B. N. Muñoz-Sánchez, S. Silva, D. Pinho, E. Vega, and R. Lima, “Generation of micro-sized pdms particles by a flow focusing technique for biomicrofluidics applications," Biomicrofluidics 10, 014122 (2016).

${ }^{46}$ C. Cohen, R. Giles, V. Sergeyeva, N. Mittal, P. Tabeling, D. Zerrouki, J. Baudry, J. Bibette, and N. Bremond, "Parallelised production of fine and calibrated emulsions by coupling flowfocusing technique and partial wetting phenomenon," Microfluidics and nanofluidics 17, 959966 (2014).

${ }^{47}$ S. Gallier, E. Lemaire, L. Lobry, and F. Peters, "Effect of confinement in wall-bounded noncolloidal suspensions,” Journal of Fluid Mechanics 799, 100-127 (2016).

${ }^{48}$ J. Eggers and E. Villermaux, "Physics of liquid jets," Reports on progress in physics 71, 036601 (2008).

${ }^{49}$ I. Johnston, D. McCluskey, C. Tan, and M. Tracey, "Mechanical characterization of bulk sylgard 184 for microfluidics and microengineering," Journal of Micromechanics and Microengineering 24, 035017 (2014).

${ }^{50}$ L. Xue, J. T. Pham, J. Iturri, and A. Del Campo, "Stick-slip friction of pdms surfaces for bioinspired adhesives," Langmuir 32, 2428-2435 (2016).

${ }^{51}$ J. D. Glover, C. E. McLaughlin, M. K. McFarland, and J. T. Pham, "Extracting uncrosslinked material from low modulus sylgard 184 and the effect on mechanical properties," Journal of Polymer Science 58, 343-351 (2020).

${ }^{52}$ A. Oláh, H. Hillborg, and G. J. Vancso, "Hydrophobic recovery of uv/ozone treated poly (dimethylsiloxane): adhesion studies by contact mechanics and mechanism of surface modification," Applied Surface Science 239, 410-423 (2005).

${ }^{53}$ F. Carrillo, S. Gupta, M. Balooch, S. J. Marshall, G. W. Marshall, L. Pruitt, and C. M. Puttlitz, "Nanoindentation of polydimethylsiloxane elastomers: Effect of crosslinking, work of adhesion, and fluid environment on elastic modulus," Journal of materials research 20, 2820-2830 (2005).

${ }^{54}$ Y. Yu, D. Sanchez, and N. Lu, "Work of adhesion/separation between soft elastomers of different mixing ratios," Journal of Materials Research 30, 2702-2712 (2015).

${ }^{55}$ P. Chin, R. L. McCullough, and W.-L. Wu, “An improved procedure for determining the work of adhesion for polymer-solid contact," The Journal of Adhesion 64, 145-160 (1997).

${ }^{56}$ S. Perutz, E. Kramer, J. Baney, C.-Y. Hui, and C. Cohen, "Investigation of adhesion hysteresis in poly (dimethylsiloxane) networks using the jkr technique," Journal of Polymer Science Part B: Polymer Physics 36, 2129-2139 (1998). 
${ }^{57}$ A. Oláh and G. J. Vancso, "Characterization of adhesion at solid surfaces: Development of an adhesion-testing device," European polymer journal 41, 2803-2823 (2005).

${ }^{58}$ M. K. Chaudhury and G. M. Whitesides, "Direct measurement of interfacial interactions between semispherical lenses and flat sheets of poly (dimethylsiloxane) and their chemical derivatives," Langmuir 7, 1013-1025 (1991).

${ }^{59}$ L. Girifalco and R. J. Good, "A theory for the estimation of surface and interfacial energies. $i$. derivation and application to interfacial tension," The Journal of Physical Chemistry 61, 904-909 (1957).

${ }^{60}$ R. J. Good and L. Girifalco, “A theory for estimation of surface and interfacial energies. iii. estimation of surface energies of solids from contact angle data," The Journal of Physical Chemistry 64, 561-565 (1960).

${ }^{61}$ F. M. Fowkes, “Attractive forces at interfaces,” Industrial \& Engineering Chemistry 56, 40-52 (1964).

${ }^{62}$ K. L. Johnson, K. Kendall, and a. Roberts, "Surface energy and the contact of elastic solids," Proceedings of the royal society of London. A. mathematical and physical sciences 324, 301313 (1971).

${ }^{63}$ M. Dirany, L. Dies, F. Restagno, L. Léger, C. Poulard, and G. Miquelard-Garnier, “Chemical modification of pdms surface without impacting the viscoelasticity: Model systems for a better understanding of elastomer/elastomer adhesion and friction," Colloids and Surfaces A: Physicochemical and Engineering Aspects 468, 174-183 (2015).

${ }^{64}$ G. A. Diaz-Quijada and D. D. Wayner, “A simple approach to micropatterning and surface modification of poly (dimethylsiloxane)," Langmuir 20, 9607-9611 (2004).

${ }^{65}$ Y. Bai, Modification of adhesion and friction by surface structuring (Lehigh University, 2014).

${ }^{66}$ A. Galliano, S. Bistac, and J. Schultz, "Adhesion and friction of pdms networks: molecular weight effects," Journal of colloid and interface science 265, 372-379 (2003).

${ }^{67}$ D. T. Nguyen, P. Paolino, M. Audry, A. Chateauminois, C. Fretigny, Y. Le Chenadec, M. Portigliatti, and E. Barthel, "Surface pressure and shear stress fields within a frictional contact on rubber," The Journal of Adhesion 87, 235-250 (2011).

${ }^{68}$ J. Bongaerts, K. Fourtouni, and J. Stokes, "Soft-tribology: lubrication in a compliant pdmspdms contact," Tribology International 40, 1531-1542 (2007).

${ }^{69}$ M. Kolli, M. Hamidouche, N. Bouaouadja, and G. Fantozzi, "Hf etching effect on sandblasted soda-lime glass properties," Journal of the European Ceramic Society 29, 2697-2704 (2009). 
${ }^{70}$ M. F. Ashby, Materials and the environment: eco-informed material choice (Elsevier, 2012).

${ }^{71}$ F. Roumili, S. Benbahouche, and J.-C. Sangleboeuf, "Mechanical strength of soda-lime glass sandblasted by gravitation," Friction 3, 65-71 (2015).

${ }^{72}$ N. Fernandez, J. Cayer-Barrioz, L. Isa, and N. D. Spencer, "Direct, robust technique for the measurement of friction between microspheres," Langmuir 31, 8809-8817 (2015).

${ }^{73}$ B. C. Donose, I. U. Vakarelski, and K. Higashitani, "Silica surfaces lubrication by hydrated cations adsorption from electrolyte solutions," Langmuir 21, 1834-1839 (2005).

${ }^{74}$ B. C. Donose, I. U. Vakarelski, E. Taran, H. Shinto, and K. Higashitani, "Specific effects of divalent cation nitrates on the nanotribology of silica surfaces," Industrial \& engineering chemistry research 45, 7035-7041 (2006).

${ }^{75}$ A. W. Chow, S. W. Sinton, J. H. Iwamiya, and T. S. Stephens, "Shear-induced particle migration in couette and parallel-plate viscometers: Nmr imaging and stress measurements," Physics of Fluids 6, 2561-2576 (1994).

${ }^{76}$ D. Merhi, E. Lemaire, G. Bossis, and F. Moukalled, "Particle migration in a concentrated suspension flowing between rotating parallel plates: Investigation of diffusion flux coefficients," Journal of Rheology 49, 1429-1448 (2005).

${ }^{77}$ G. Davies and J. Stokes, "Thin film and high shear rheology of multiphase complex fluids," Journal of Non-newtonian fluid mechanics 148, 73-87 (2008).

${ }^{78}$ K. Yeo and M. R. Maxey, "Dynamics of concentrated suspensions of non-colloidal particles in couette flow," Journal of Fluid Mechanics 649, 205 (2010).

${ }^{79}$ K. Yeo and M. R. Maxey, "Ordering transition of non-brownian suspensions in confined steady shear flow," Physical Review E 81, 051502 (2010).

${ }^{80}$ I. E. Zarraga, D. A. Hill, and D. T. Leighton Jr, "The characterization of the total stress of concentrated suspensions of noncolloidal spheres in newtonian fluids," Journal of Rheology 44, 185-220 (2000).

${ }^{81}$ S. H. Maron and P. E. Pierce, “Application of ree-eyring generalized flow theory to suspensions of spherical particles," Journal of colloid science 11, 80-95 (1956).

${ }^{82}$ A. Gans, O. Pouliquen, and M. Nicolas, “Cohesion-controlled granular material,” Physical Review E 101, 032904 (2020).

${ }^{83}$ S. Mandal, M. Nicolas, and O. Pouliquen, "Rheology of cohesive granular media: Shear banding, hysteresis, and nonlocal effects," Physical Review X 11, 021017 (2021).

${ }^{84} \mathrm{C}$. Martin and R. Bordia, "Influence of adhesion and friction on the geometry of packings of 
spherical particles," Physical Review E 77, 031307 (2008).

${ }^{85}$ C. Dominik and A. Tielens, "Resistance to rolling in the adhesive contact of two elastic spheres," Philosophical Magazine A 72, 783-803 (1995).

${ }^{86}$ S. Krijt, C. Dominik, and A. Tielens, "Rolling friction of adhesive microspheres," Journal of Physics D: Applied Physics 47, 175302 (2014).

${ }^{87}$ P. Silberzan, S. Perutz, E. J. Kramer, and M. K. Chaudhury, "Study of the self-adhesion hysteresis of a siloxane elastomer using the jkr method," Langmuir 10, 2466-2470 (1994).

${ }^{88}$ G. Y. Choi, S. Kim, and A. Ulman, "Adhesion hysteresis studies of extracted poly (dimethylsiloxane) using contact mechanics," Langmuir 13, 6333-6338 (1997).

${ }^{89}$ S. Kim, G. Y. Choi, A. Ulman, and C. Fleischer, "Effect of chemical functionality on adhesion hysteresis," Langmuir 13, 6850-6856 (1997).

${ }^{90}$ H. She, D. Malotky, and M. K. Chaudhury, "Estimation of adhesion hysteresis at polymer/oxide interfaces using rolling contact mechanics," Langmuir 14, 3090-3100 (1998).

${ }^{91}$ A. Singh, C. Ness, R. Seto, J. J. de Pablo, and H. M. Jaeger, "Shear thickening and jamming of dense suspensions: The "roll” of friction,” Physical Review Letters 124, 248005 (2020). 